\title{
Association between coffee or caffeine consumption and fecundity and fertility: a systematic review and dose-response meta-analysis
}

This article was published in the following Dove Press journal: Clinical Epidemiology

\author{
Julie Lyngsø' \\ Cecilia Høst \\ Ramlau-Hansen' \\ Bjørn Bay ${ }^{2}$ \\ Hans Jakob Ingerslev ${ }^{3}$ \\ Adam Hulman ${ }^{1,4}$ \\ Ulrik Schiøler Kesmodel ${ }^{5}$ \\ 'Department of Public Health, \\ Section for Epidemiology, Aarhus \\ University, Aarhus, ${ }^{2}$ The Fertility \\ Clinic, Regional Horsens Hospital, \\ Horsens, ${ }^{3}$ Department of Obstetrics \\ and Gynaecology, Aarhus University \\ Hospital, Aarhus, ${ }^{4}$ Danish Diabetes \\ Academy, Odense, ${ }^{5}$ Department of \\ Obstetrics and Gynaecology, Herlev \\ and Gentofte Hospital, Herlev, \\ Denmark
}

Objective: The aim was to investigate whether coffee or caffeine consumption is associated with reproductive endpoints among women with natural fertility (ie, time to pregnancy [TTP] and spontaneous abortion [SAB] ) and among women in fertility treatment (ie, clinical pregnancy rate or live birth rate).

Design: This study was a systematic review and dose-response meta-analysis including data from case-control and cohort studies.

Methods: An extensive literature search was conducted in MEDLINE and Embase, with no time and language restrictions. Also, reference lists were searched manually. Two independent reviewers assessed the manuscript quality using the Newcastle-Ottawa Scale (NOS). A twostage dose-response meta-analysis was applied to assess a potential association between coffee/ caffeine consumption and the outcomes: TTP, SAB, clinical pregnancy, and live birth. Heterogeneity between studies was assessed using Cochrane $Q$-test and $I^{2}$ statistics. Publication bias was assessed using Egger's regression test.

Results: The pooled results showed that coffee/caffeine consumption is associated with a significantly increased risk of SAB for $300 \mathrm{mg}$ caffeine/day (relative risk [RR]: 1.37, 95\% confidence interval [95\% CI]: $1.19 ; 1.57$ ) and for $600 \mathrm{mg}$ caffeine/day (RR: $2.32,95 \% \mathrm{CI}: 1.62$; 3.31). No association was found between coffee/caffeine consumption and outcomes of fertility treatment (based on two studies). No clear association was found between exposure to coffee/ caffeine and natural fertility as measured by fecundability odds ratio (based on three studies) or waiting TTP (based on two studies).

Conclusion: Results from this meta-analysis support the growing evidence of an association between coffee/caffeine intake and the risk of SAB. However, viewing the reproductive capacity in a broader perspective, there seems to be little, if any, association between coffee/caffeine consumption and fecundity. In general, results from this study are supportive of a precautionary principle advised by health organizations such as European Food Safety Authority (EFSA) and World Health Organization (WHO), although the advised limit of a maximum of two to three cups of coffee/200-300 mg caffeine per day may be too high.

Keywords: coffee, caffeine, fecundity, fertility, spontaneous abortion, assisted reproduction

\section{Plain language summary}

Coffee and other caffeinated beverages are widely consumed by women desiring to conceive as well as pregnant women. Whether such consumption reduces the chance of achieving a pregnancy or may have harmful effects on the developing fetus is widely debated. As a contribution to this debate and given the growing concern of subfecundity and infertility in public health, 
we investigated the risk of coffee or caffeine consumption on the ability to conceive a pregnancy and to carry the pregnancy to term. Results from this study showed an increased risk of experiencing a spontaneous abortion with increasing consumption of coffee/ caffeine during early pregnancy. No clear association was found between coffee/caffeine consumption and the chance of achieving a pregnancy among women/couples trying to conceive naturally or by women/couples receiving fertility treatment. Results from this study support the precautionary principle advised by health organizations such as European Food Safety Authority (EFSA) and World Health Organization (WHO), although the advised limit of a maximum of two to three cups of coffee/200-300 mg caffeine per day may be too high.

\section{Introduction}

Subfecundity and infertility are multifactorial conditions of growing concern in public health. Worldwide, one in six couples experience fecundity problems during their reproductive lifetime. ${ }^{1-3}$ While the cause often may be explained by failure of ovulation, damages on the fallopian tubes, or low sperm count, the reduced fecundity is still unexplained in many cases. Thus, investigations of a potential effect of modifiable exposures on longer waiting time to pregnancy (TTP), increased rate of spontaneous abortion (SAB), or reduced live birth rate are important.

Coffee consumption is common among people throughout the world. Worldwide, Finland has the highest consumption (12.0 kg coffee/person/year), with Denmark being the fourth most coffee-consuming country $(8.7 \mathrm{~kg}$ coffee/person/ year). Also, coffee and other caffeine-containing drinks and foods are widely consumed by women desiring to conceive and by pregnant women. On average, a cup of coffee contains $\sim 100 \mathrm{mg}$ of caffeine. ${ }^{4}$

Even small effects of coffee or caffeine intake on fecundity could have considerable public health consequences, considering the large consumption of these substances.

The European Food Safety Authority (EFSA) recommends women desiring to conceive and pregnant women to keep their daily caffeine consumption below $200 \mathrm{mg},{ }^{5}$ while WHO recommends a daily caffeine intake below $300 \mathrm{mg} .{ }^{6}$ Caffeine is a central ingredient in coffee, while other sources of caffeine include tea, soft drinks, chocolate, and some medications.

The association between caffeine intake and female fecundity has been studied with inconsistent findings, in studies with both retrospectively ${ }^{7-13}$ and prospectively collected exposure data. ${ }^{14-19} \mathrm{~A}$ high level of coffee consumption during pregnancy has been associated with an increased risk of fetal death after 20 weeks of gestation ${ }^{20,21}$ and stillbirth. ${ }^{22}$ In meta-analyses, an increased risk of low birth weight and small for gestational age was found, ${ }^{23}$ but no association was found between caffeine intake during pregnancy and the risk of preterm birth. ${ }^{23,24}$

Recently, a meta-analysis reported that an increment in intake of $100 \mathrm{mg}$ caffeine per day was associated with a $14 \%$ increased risk of SAB. ${ }^{23}$ However, the authors did not account for the varying quality of the included studies, including varying (or none) adjustment for potential confounders. Studies that fail to adjust for important risk factors associated with SAB may over- or underestimate the risk associated with caffeine consumption. Hence, taking adjustment status into account would considerably improve the quality of a meta-analysis. Moreover, other substances in coffee besides caffeine might play an important role. Finally, applying a broader view on fecundity through inclusion of different reproductive endpoints among both women conceiving naturally and following fertility treatment could extend the use of the results and help general practitioners and fertility clinics provide balanced information to women and couples seeking advice or even fertility treatment. No meta-analysis has previously reported on a possible association between coffee/caffeine intake and TTP or endpoints among couples in fertility treatment.

The aim of this systematic review and dose-response meta-analysis was to investigate whether consumption of coffee or caffeine is associated with reproductive endpoints among women with natural fertility (ie TTP and SAB) and among women in fertility treatment (ie clinical pregnancy rate and live birth rate).

\section{Methods}

A review protocol is available from the PROSPERO database. ${ }^{25}$ The protocol was registered on June 9, 2015 (registration number: CRD42015020333).

\section{Search strategy and selection}

We conducted a comprehensive literature search in MEDLINE and Embase databases with the help of a medical librarian on May 26, 2015. The following keywords and medical subject headings were used to identify relevant articles in the databases: (["Fertility" or "Infertility, Female" or "Reproduction" or "Preconception Care") or ["Reproductive Techniques, Assisted" or "Reproductive Techniques" or "fertility treatment"]) AND ("Caffeine/adverse effects" or "Coffee") AND ("Case-Control Studies" or "Cohort Studies"). To include non-indexed new literature, an additional search was performed using free text terms. No restrictions were made regarding study language or year of publication. Further, the reference lists of retrieved articles and reviews were searched manually for additionally relevant studies. The 
first author reviewed all retrieved articles for eligibility by title and abstract by consulting with at least one co-author in cases of any doubt about the inclusion of a given study or not. Potentially eligible articles were thoroughly read in full text to check whether they fulfilled the following inclusion criteria:

1. Participants had to be either women/couples trying to conceive naturally or women/couples trying to conceive by medically assisted reproduction (MAR) treatment

2. Studies that reported a numerical exposure range of either coffee or caffeine consumption

3. Studies that investigated at least one of the outcomes of interest: TTP, SAB, clinical pregnancy, and live birth

4. Articles that reported data from original studies (ie, no review articles)

5. Studies of which the design was a case-control study or a cohort study (ie, no RCT)

Before finalizing the manuscript, the database search was repeated on April 3, 2017.

\section{Data extraction}

All included full text articles were read independently by at least two authors. A data extraction form was developed a priori, ensuring a standardized procedure. The following data were extracted: lead author surname, study title, country of origin, year of publication and journal, study design, study population (size and selection), exposure and outcome assessment, covariates adjusted for, main results, study limitations, and assessment of quality scoring.

We assessed the quality of all included studies using the Newcastle-Ottawa Scale (NOS). ${ }^{26}$ Thus, the selection process and comparability of the study groups as well as the ascertainment of exposure or outcome of interest of the included studies were evaluated for either cohort or case-control studies (please see scoring details in the Supplementary material). In addition to the NOS scheme, we constructed an explanatory form ensuring a standardized scoring among the authors with regard to the specific scope of this review (Supplementary material). When using NOS to evaluate study comparability, one has to choose the two most important covariates. Hence, based on the current knowledge, maternal age was chosen as the most important factor to adjust for. As additionally important adjustment factors, smoking was chosen for studies evaluating TTP and MAR treatment, while maternal alcohol consumption was chosen for those investigating SAB. All studies were allocated a total score between 0 and 9 independently by a minimum of two authors. Any inconsistency was resolved by discussion, leading to a uniform decision. Studies with a total score of $\geq 7$ were considered as "high-quality studies", and in a sub-analysis, studies were stratified according to this selected cut-off value. Regardless of total NOS score, all studies were included in the review.

This review is reported in accordance with the PRISMA statement ${ }^{27}$ for reporting of systematic review and metaanalysis. Supplementary material regarding the full search strategy for MEDLINE, elaborated reasons for exclusion, and NOS explanatory form are all available online.

\section{Outcomes}

The ability to naturally conceive was investigated by two broad fecundability measures including TTP (ie, the number of months or cycles taken by a couple to conceive, when being sexually active and not using birth control) and fecundability odds ratio (FOR) (ie, the ratio of couple's probability of conceiving in one menstrual cycle). ${ }^{28}$ The capability of maintaining a pregnancy after conception was evaluated by the risk of experiencing an SAB (yes/no). Among couples receiving MAR treatment, a possible association was investigated using different endpoints to evaluate a successful fertility treatment (ie, achieving a pregnancy or live birth after fertility treatment; yes/no).

\section{Statistical analysis}

Although still included in the review, studies were excluded from the meta-analyses if they did not provide information on the number of cases and total number of subjects within each exposure category and whose corresponding author did not respond to emails asking for additional data to calculate these numbers. ${ }^{7,9,10,14,15,17,29-33}$ Also, we excluded studies that did not report on three or more quantitative categories of coffee/caffeine consumption, ${ }^{34-37}$ as these cannot contribute sufficient data when performing a dose-response meta-analysis. ${ }^{38}$

We applied a two-stage dose-response meta-analysis to assess a potential association between coffee/caffeine consumption and our outcomes. ${ }^{39}$ For fecundability and MAR treatment, we included only fixed effects in the metaanalysis due to the low number of included studies, ${ }^{40}$ while for SAB, we conducted a random-effects meta-analysis after considering heterogeneity between studies. As coffee/caffeine consumption was reported on various scales, we converted exposure data into a uniform measurement (mg/day). Studies reporting consumption in cups of coffee were recalculated into an approximate caffeine content, assuming that one cup of coffee in general contains $100 \mathrm{mg}$ caffeine., ${ }^{4,41}$ As the approximation of caffeine content per cup of coffee might be imprecise, a sensitivity analysis was performed, assuming 
that one cup of coffee contained $150 \mathrm{mg}$ caffeine. Coffee/ caffeine consumption was modeled using restricted cubic splines with three knots at the 10th, 50th, and 90th percentiles of the distribution of doses. ${ }^{38,42,43}$ For each model, we present an overall $p$-value testing that the two regression coefficients describing the nonlinear relationship were simultaneously equal to zero. Also, upon finding a statistical significant association, we present a $p$-value testing the linear versus nonlinear relationship. ${ }^{44}$ When analyzing risk of SAB, we performed stratified analyses by type of consumption (caffeine vs coffee), adjustment status (crude vs any adjustment), study quality (NOS $>7$ vs NOS $<7$ ), and study design (cohort vs case-control). Similar analyses were not possible for other outcomes due to the low number of studies. Heterogeneity between studies was assessed using the Cochrane $Q$-test and the $I^{2}$ statistic. Low heterogeneity was defined by $I^{2}$ values $<25 \%$, while a $I^{2}$ value $>75 \%$ was considered an indicator of substantial heterogeneity. ${ }^{45}$ Publication bias was assessed for SAB studies, using Egger's regression test. ${ }^{46}$ As meta-analysis for fecundity and MAR endpoints only included a maximum of three studies, tests of heterogeneity and publication bias were irrelevant for these outcomes. ${ }^{47}$ All statistical analyses were performed using the "dosresmeta" and "metafor" packages in $\mathrm{R}$ version 3.3.1. ${ }^{48,49} p$-values $<0.05$ were considered statistically significant.

\section{Results}

\section{Characteristics of the included studies}

A total number of 379 articles were identified through the systematic literature search, and an additional 68 articles were identified through a manual search of their references (Figure 1). Of the total 447 articles, 136 were duplicates and thus excluded. The remaining 311 unique articles were screened by title and, if necessary, by abstract, and $195 \mathrm{did}$ not meet the eligibility criteria. Full-texts were assessed for the remaining 116 articles. Of these, 68 articles were excluded due to not meeting the eligibility criteria, and one article was excluded due to insufficient data reporting.

Thus, a total of 47 studies were included in the systematic review. Of these, 35 studies had dose-response information (ie, reporting at least three or more quantitative exposure categories) and were included in our meta-analyses. Of these 35 studies, 5 studies reported about the endpoints of fecundity $9,10,16,19,50,27$ studies reported about $\mathrm{SAB},{ }^{21,32,34,51-73}$ and 3 studies reported on the outcomes of MAR ${ }^{74-76}$ Results from the remaining 12 studies that did not provide sufficient data to be included in the meta-analysis are included as a narrative description.
All included studies were published between 1988 and 2015. Thirty-one were cohort studies, while 16 were case-control studies. Study characteristics, results, and the assigned total NOS scores are presented in Tables 1-3 (for NOS elaborations, please see the Table S1). The presented results cover a variety of countries with large differences in exposure levels ranging from non-consumers to those who consume over $1,200 \mathrm{mg} /$ day (corresponding to 12 cups of coffee/day). Also, adjustment for potential confounders varied considerably between studies (Tables 1-3).

\section{FOR}

In the three cohort studies providing sufficient data to be included in the meta-analysis, we found no indication of an association between caffeine intake up to $400 \mathrm{mg}$ /day and altered FOR (overall $p=0.76$; Figure 2A). Compared with no caffeine intake, the pooled odds ratios for altered fecundability were 1.03 (95\% confidence interval [CI]: $0.83 ; 1.28)$ for $100 \mathrm{mg}$ caffeine/day and 1.09 (95\% CI: 0.86; 1.37) for $400 \mathrm{mg}$ caffeine/day.

\section{Studies not eligible for meta-analysis}

Five other studies have addressed a possible association between coffee/caffeine consumption and fecundability with contradicting findings. Two studies from the US ${ }^{13,15}$ including a total of 104 and 6,303 women, respectively reported reduced fecundability with higher caffeine consumption in the late 1980s. However, results by three later studies (two European studies and one US study) ${ }^{14,17,30}$ including 259 and 423 , and 470 women, respectively, support the findings of no association between caffeine consumption on fecundability.

\section{TTP}

Only two cohort studies provided sufficient data on TTP, to be included in the dose-response meta-analysis. The results did not indicate an association ( $p=0.43$ for TTP $>6$ months and $p=0.98$ for TTP $>12$ months) (Figure $2 \mathrm{~B}$ and $2 \mathrm{C}$ ).

Compared with no caffeine intake, the pooled relative risks for TTP $>6$ months were 0.92 (95\% CI: 0.79 ; 1.07) for $100 \mathrm{mg}$ caffeine/day and 0.76 (95\% CI: $0.49 ; 1.18)$ for 600 mg caffeine/day. For TTP $>12$ months, the pooled relative risks were $0.99(95 \% \mathrm{CI}: 0.88 ; 1.11)$ for $100 \mathrm{mg}$ caffeine/ day and 0.97 (95\% CI: $0.71 ; 1.33)$ for $600 \mathrm{mg}$ caffeine/day, compared with no caffeine intake.

\section{Studies not eligible for meta-analysis}

Two studies that were not included in the meta-analysis have investigated a possible association between caffeine 


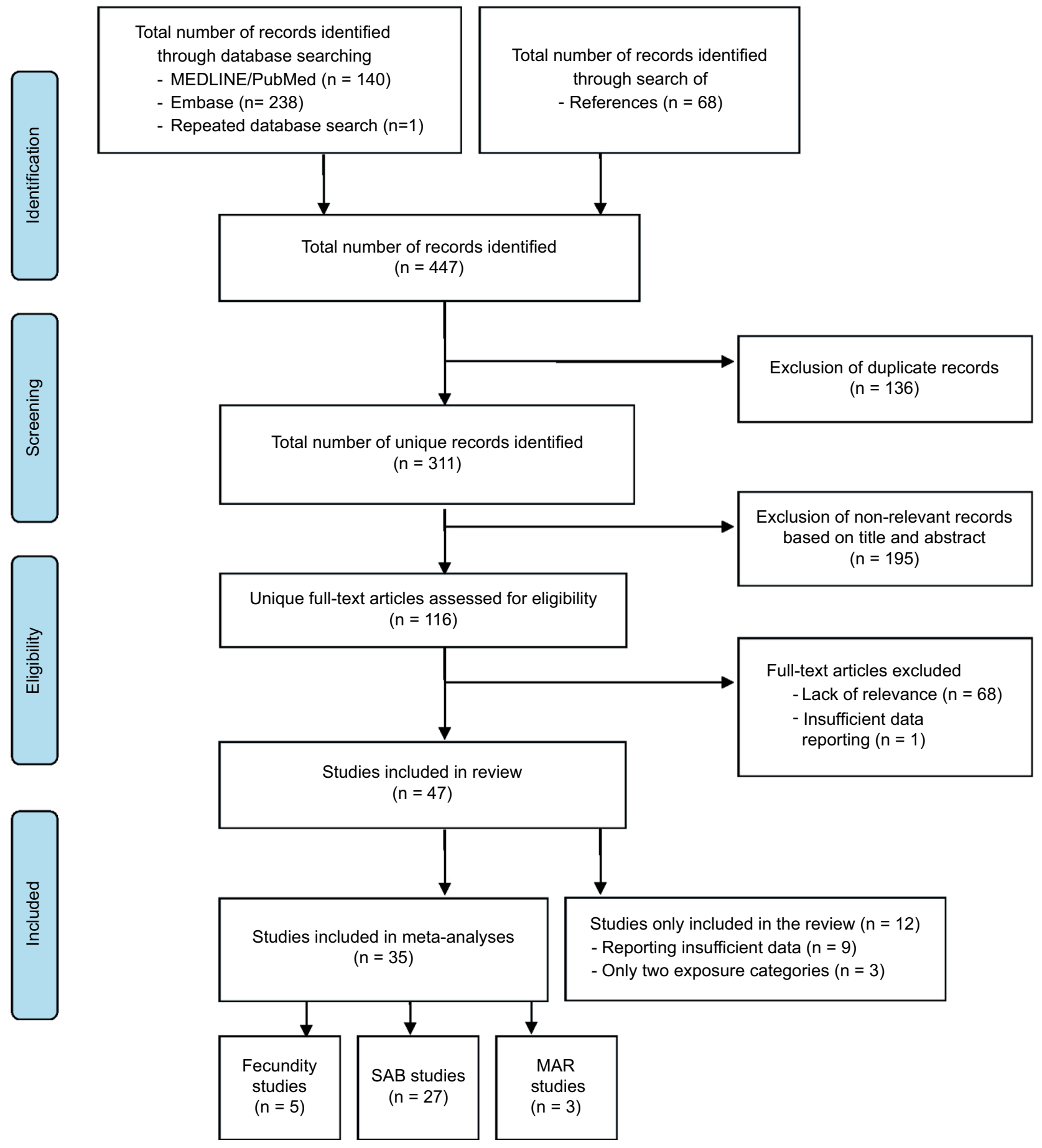

Figure I Flow diagram.

Abbreviations: SAB, spontaneous abortion; MAR, medically assisted reproduction.

consumption and TTP. Bolúmar et $\mathrm{al}^{7}$ reported on 3,146 European women and found an increased risk of TTP $>9.5$ months with a caffeine intake of $>500 \mathrm{mg} /$ day when reporting retrospectively on their first pregnancy. However, no association was found when reporting on the most recent waiting time. Another study including 66 American women ${ }^{29}$ did not find an association between caffeine consumption and TTP.

\section{$S A B$}

We found that higher intake of caffeine was associated with higher risk of spontaneous abortion (overall association $p<0.0001 ; p$ for non-linearity $<0.01$ ), based on 27 studies providing sufficient data to be included in the meta-analysis (Figure 2D). Compared with no caffeine intake, the pooled relative risks for $\mathrm{SAB}$ were 1.08 (95\% CI: $1.03 ; 1.13)$ for 
Table I Characteristics and results of publications on coffee/caffeine and fecundity endpoints

\begin{tabular}{|c|c|c|c|c|c|}
\hline $\begin{array}{l}\text { Author and } \\
\text { year }\end{array}$ & Country & Design & $\begin{array}{l}\text { Study groups } \\
\text { included in the analyses }\end{array}$ & $\begin{array}{l}\text { Source of } \\
\text { caffeine }\end{array}$ & $\begin{array}{l}\text { Controls for } \\
\text { maternal age }\end{array}$ \\
\hline
\end{tabular}

Wilcox et al,

$1988^{15}$

Christianson et al, US $1989^{13}$

Olsen, 1991

Denmark

Cohort

$\mathrm{N}=5,309$ non-smoking pregnant Danish women who had reached their 3 rd trimester

Cases: 523

Florack et al, The

$1994^{17}$

Netherlands

Cohort

$\mathrm{N}=259$

Coffee

Dutch women planning a pregnancy within a year, hospital workers

Alderete et al, US $1995^{10}$

US

Cohort

$\mathrm{N}=787$

Primigravidas and married American women

European women in the fertile age, planning their first
$\mathrm{N}=3,146$

pregnancy

Cohort

Spain
Coffee $1997^{7}$

Caan et al, $1998^{16}$

US

US

Hakim et al,

$1998^{19}$

Jensen et al, $1998^{14}$
Cohort

$\mathrm{N}=187$ volunteering American women, pregnancy
planners who had been trying for 3 months or less conceive

In total 737 cycles; II 5 pregnant cycles and 622 nonpregnant cycles

Cohort

$\mathrm{N}=98$ volunteering American women in the

reproductive age, employed at two semiconductor plants

No. of conceptions: 90

No. of menstrual cycles: 536

$\mathrm{N}=423$ volunteering Danish couples, nulliparous and

pregnancy planners with I,596 cycles

Multiple Yes

No

Multiple Yes

Yes




\begin{tabular}{|c|c|c|c|}
\hline $\begin{array}{l}\text { Controls } \\
\text { for other } \\
\text { important } \\
\text { covariates }\end{array}$ & Confounding factors & Main results & $\begin{array}{l}\text { Total NOS } \\
\text { score }\end{array}$ \\
\hline Yes & $\begin{array}{l}\text { Age, frequency of intercourse, age at menarche, } \\
\text { woman's prenatal exposure to mother's smoking. } \\
\text { Subanalysis resticted to non-smokers }\end{array}$ & $\begin{array}{l}\text { Women with a caffeine intake had a decreased fecundability } \\
\text { FOR: } 0.5 \mathrm{I}(95 \% \mathrm{Cl} 0.35 ; 0.75)\end{array}$ & 6 \\
\hline Yes & Ethnicity, parity, smoking & $\begin{array}{l}\text { Women with a coffee intake of }>7 \text { cups/day had an increased } \\
\text { risk of experiencing difficulties in becoming pregnant } \\
\text { RR: I.96* }\end{array}$ & 4 \\
\hline Yes & $\begin{array}{l}\text { Main analysis - data stratified on smoking status. } \\
\text { Adjusted for number of pregnancies, father's age, } \\
\text { mother's and father's alcohol drinking habits, shift } \\
\text { work, mother's education, mother's and father's } \\
\text { smoking habits }\end{array}$ & $\begin{array}{l}\text { Among nonsmokers, there was no association between } \\
\text { coffee/tea intake }>8 \text { cups/day and TTP }>6 \text { or TTP }>12 \\
\text { months } \\
\text { OR: } 0.92 \text { ( } 95 \% \mathrm{Cl} 0.7 \text { I; } 1.19) \text { and OR: } 0.98 \text { ( } 95 \% \mathrm{Cl} 0.70 ; 1.37) \\
\text { Among smokers, an intake of }>8 \text { cups/day was associated } \\
\text { with TTP }>12 \text { months } \\
\text { OR: } 1.35(95 \% \mathrm{Cl} \text { I.02; I.48) }\end{array}$ & 6 \\
\hline No & $\begin{array}{l}\text { Main analysis = crude analysis. } \\
\text { Subanalysis: controling for smoking and partners } \\
\text { behavioral risk factors }\end{array}$ & $\begin{array}{l}\text { Women with a caffeine intake of } 400-700 \mathrm{mg} / \text { day had an } \\
\text { increased fecundability } \\
\text { FOR: } 2.10(95 \% \mathrm{CI} \quad \text { I.20; } 3.70)\end{array}$ & 7 \\
\hline Yes & $\begin{array}{l}\text { Stratification by smoking status. } \\
\text { Adjusted for age, race, BMl, education, prior use of } \\
\text { contraceptive pills, alcohol consumption, husband's } \\
\text { smoking habits, income, whether pregnancy was } \\
\text { planned, abnormal reproductive conditions, immune } \\
\text { and endocrine disorders }\end{array}$ & $\begin{array}{l}\text { Nonsmoking women with a coffee intake }>3 \text { cups/day had no } \\
\text { increased risk of TTP }>3 \text { months }(187 \text { cases }) \\
\text { OR: } 1.10(95 \% \mathrm{Cl} 0.70 ; 1.80) \\
\text { TTP }>6 \text { months }(104 \text { cases }) \\
\text { OR: } 1.00(95 \% \mathrm{Cl} 0.60 ; 1.80) \text { or TTP }>12 \text { months ( } 56 \text { cases) } \\
\text { OR: } 1.00(95 \% \mathrm{Cl} 0.50 ; 1.70)\end{array}$ & 7 \\
\hline Yes & $\begin{array}{l}\text { Age, parity, smoking, alcohol consumption, } \\
\text { frequency of intercourse, educational level, working } \\
\text { status, use of oral contraceptives, and country }\end{array}$ & $\begin{array}{l}\text { Women with a caffeine intake during the first pregnancy of } \\
>500 \mathrm{mg} / \text { day had an increased risk of TTP }>9.5 \text { months } \\
\text { OR: I. } 45(95 \% \mathrm{Cl} \mathrm{I.03;} \mathrm{2.04)} \\
\text { No association between total caffeine intake }>500 \mathrm{mg} / \text { day or } \\
\text { coffee intake }>5 \text { cups/day and TTP }>9.5 \text { months }(\mathrm{N}=3.053) \\
\text { OR: } 1.32(95 \% \mathrm{Cl} 0.94 ; 1.86) \text { and OR: } 1.26(95 \% \mathrm{Cl} 0.91 \text {; } \\
\text { I.74) during the most recent waiting time }\end{array}$ & 6 \\
\hline Yes & $\begin{array}{l}\text { Age, BMI, gravidity, frequency of intercourse, } \\
\text { smoking, and alcoholic beverage }\end{array}$ & $\begin{array}{l}\text { No risk of reduced fecundability with total caffeine intake } \\
>106.8 \mathrm{mg} / \text { day } \\
\text { OR: I.09 }(95 \% \mathrm{Cl} 0.63 ; \text { I.89) } \\
\text { or coffee intake >I cup/day } \\
\text { OR: } 0.77(95 \% \mathrm{Cl} 0.43 ; \text { I.37) }\end{array}$ & 6 \\
\hline Yes & $\begin{array}{l}\text { Participants' age, number of occurrences of sexual } \\
\text { intercourse in each month, alcohol consumption. } \\
\text { The analysis was restricted to nonsmoking women }\end{array}$ & $\begin{array}{l}\text { Nonsmoking women had no risk of reduced fecundability } \\
\text { with caffeine intake }>300 \mathrm{mg} / \text { day } \\
\text { OR: } 0.83(95 \% \mathrm{Cl} 0.34 ; 2.0 \mathrm{l})\end{array}$ & 7 \\
\hline Yes & $\begin{array}{l}\text { Stratification by smoking. } \\
\text { Total caffeine adjusted for female BMI, alcohol } \\
\text { intake, deseases of the reproductive organs, semen } \\
\text { quality, duration of menstrual cycle }\end{array}$ & $\begin{array}{l}\text { Nonsmoking women had no risk of reduced fecundability } \\
\text { with total caffeine intake }>700 \mathrm{mg} / \text { day } \\
\text { FOR: } 0.63(95 \% \mathrm{Cl} 0.25 ; 1.60) \text { or caffeine intake from coffee } \\
>300 \mathrm{mg} / \text { day } \\
\text { FOR: } 0.87(95 \% \mathrm{Cl} 0.32 ; 2.37) \\
\text { Among smokers, a total intake of caffeine } 0-299 \mathrm{mg} / \text { day and } \\
\text { a caffeine intake from coffee }>300 \mathrm{mg} / \text { day were associated } \\
\text { with reduced fecundability (FOR: } 0.55 \text { [ } 95 \% \mathrm{Cl} 0.32 ; 0.98] \\
\text { and FOR: } 0.34[95 \% \mathrm{Cl} 0.12 ; 0.98] \text {, respectively) }\end{array}$ & 5 \\
\hline Yes & $\begin{array}{l}\text { Age, average alcohol consumption and cigarette } \\
\text { consumption per standardized 28-day cycle, and } \\
\text { prior history of SAB }\end{array}$ & $\begin{array}{l}\text { No risk of longer TTP with total caffeine intake (RR: } 1.00 \\
[95 \% \mathrm{Cl} 0.99 ; 1.01])\end{array}$ & 7 \\
\hline
\end{tabular}


Table I (Continued)

\begin{tabular}{llll}
\hline $\begin{array}{l}\text { Author and } \\
\text { year }\end{array}$ & Country & Design & Study groups \\
included in the analyses & Controls for & Source of \\
caffeine & & maternal age
\end{tabular}

\begin{tabular}{lll}
\hline $\begin{array}{l}\text { Taylor et al, } \\
2011^{30}\end{array}$ & Cohort & $\begin{array}{l}\mathrm{N}=470 \text { American women who were sexually active and Multiple } \\
\text { with no history of infertility }\end{array}$ \\
$\begin{array}{l}\text { Hatch et al, } \\
2012^{50}\end{array}$ & Cohort & $\begin{array}{l}\mathrm{N}=2,484 \text { Danish women, planning a pregnancy who had Multiple/ } \\
\text { not been trying to conceive for }>6 \text { cycles at study entry coffee } \\
\end{array}$ \\
& $\begin{array}{l}\text { No. of pregnancies: } 2,484 \\
\text { Cycles at risk: } 15,225\end{array}$
\end{tabular}

Notes: *No $95 \% \mathrm{Cl}$ provided.

Abbreviations: $95 \% \mathrm{Cl}, 95 \%$ confidence interval; FOR, fecundability odds ratio; NOS, Newcastle-Ottawa scale; TTP, time to pregnancy; SAB, spontaneous abortion; $\mathrm{BMI}$, body mass index.

Table 2 Characteristics and results of publications on coffee/caffeine and spontaneous abortion (SAB)

\begin{tabular}{|c|c|c|c|c|c|}
\hline Author and year & Country & Design & Study groups included in the analyses & $\begin{array}{l}\text { Source of } \\
\text { caffeine }\end{array}$ & $\begin{array}{l}\text { Controls for } \\
\text { maternal age }\end{array}$ \\
\hline $\begin{array}{l}\text { Srisuphan and } \\
\text { Bracken, } 1986^{34}\end{array}$ & US & Cohort & $\begin{array}{l}\mathrm{N}=2,188 \text { pregnant American women attending } \\
\text { care at } 29 \text { private hospitals } \\
\text { Cases: } 345\end{array}$ & Multiple & Yes \\
\hline $\begin{array}{l}\text { Axelsson and } \\
\text { Rylander, } 1989^{31}\end{array}$ & Sweden & Cohort & $\begin{array}{l}N=654 \text { Swedish shift-working women, including } \\
970 \text { pregnancies }\end{array}$ & Coffee & Yes \\
\hline Wilcox et al, $1990^{66}$ & US & Cohort & $\begin{array}{l}\mathrm{N}=104 \text { healthy volunteering American women, } \\
\text { who did not become pregnant in the first } 3 \\
\text { months of trying to conceive with } 171 \text { pregnancies } \\
\text { Cases: } 43\end{array}$ & Multiple & Yes \\
\hline Fenster et al, $\left.199\right|^{51}$ & US & $\begin{array}{l}\text { Case- } \\
\text { control }\end{array}$ & $\begin{array}{l}\mathrm{N}=I, 89 \mathrm{I} \\
\text { Cases: } 607 \text { American women with } \mathrm{SAB} \text { within the } \\
\text { first } 20 \text { weeks of gestation } \\
\text { Controls: I,284 women, frequency matched by } \\
\text { last menstrual period and hospital, selected two } \\
\text { per case from county live births }\end{array}$ & Multiple & Yes \\
\hline Kline et al, $1991^{60}$ & US & $\begin{array}{l}\text { Case- } \\
\text { control }\end{array}$ & $\begin{array}{l}\mathrm{N}=2,44 \text { I } \\
\text { Cases: I, I } 35 \text { American women with SAB within } \\
\text { the first } 28 \text { weeks of gestation } \\
\text { Controls: I,306 pregnant women, matched by age } \\
\text { and payment group }\end{array}$ & Multiple & Yes \\
\hline Parazzini et al, $|99|^{36}$ & Italy & $\begin{array}{l}\text { Case- } \\
\text { control }\end{array}$ & $\begin{array}{l}\mathrm{N}=260 \\
\text { Cases: } 94 \text { Italian women with two or more } \\
\text { unexplained miscarriages and no full-term pregnancy } \\
\text { Controls: } 176 \text { women with normal delivery }\end{array}$ & Coffee & Yes \\
\hline $\begin{array}{l}\text { Armstrong et al, } \\
1992^{53}\end{array}$ & US & Cohort & $\begin{array}{l}\mathrm{N}=35,848 \text { American women with previous } \\
\text { pregnancy ending in either delivery or SAB } \\
\text { Cases: } 7,606\end{array}$ & Coffee & Yes \\
\hline $\begin{array}{l}\text { Infante-Rivard et al, } \\
\mid 993^{61}\end{array}$ & Canada & $\begin{array}{l}\text { Case- } \\
\text { control }\end{array}$ & $\begin{array}{l}\mathrm{N}=\mathrm{I}, 323 \\
\text { Cases: } 33 \text { I Canadian women admitted for fetal } \\
\text { loss } \\
\text { Controls: } \mathrm{N}=992 \text { pregnant women expected } \\
\text { to deliver at the same hospital, matched } 3: \mathrm{I} \text { on } \\
\text { periods of pregnancy }\end{array}$ & Coffee & Yes \\
\hline
\end{tabular}




\begin{tabular}{|c|c|c|c|}
\hline $\begin{array}{l}\text { Controls } \\
\text { for other } \\
\text { important } \\
\text { covariates }\end{array}$ & Confounding factors & Main results & $\begin{array}{l}\text { Total NOS } \\
\text { score }\end{array}$ \\
\hline Yes & $\begin{array}{l}\text { Age, trying to get pregnant, frequency of } \\
\text { unprotected intercourse, BMI, average alcohol } \\
\text { intake, smoking }\end{array}$ & $\begin{array}{l}\text { No risk of reduced fecundability among women with a } \\
\text { caffeine intake }>300 \mathrm{mg} / \text { day (FOR: } 0.89 \text { [95\% Cl } 0.58 ; 1.38] \text { ) }\end{array}$ & 6 \\
\hline Yes & $\begin{array}{l}\text { Age, partner's age, BMI, pack-years of smoking, } \\
\text { number of alcoholic beverages consumed per week, } \\
\text { physical activity (METs/week), and frequency of } \\
\text { intercourse } \\
\text { (also adjusted for tea and cola in analyses concerning } \\
\text { coffee) }\end{array}$ & $\begin{array}{l}\text { No risk of reduced fecundability among women with a } \\
\text { caffeine intake }>300 \mathrm{mg} / \text { day (FOR: I.06 [95\% Cl } 0.92 ; 1.23] \text { ) } \\
\text { or coffee intake }>3 \text { cups/day (FOR: } 1.01 \text { I }[95 \% \mathrm{Cl} 0.80 ; 1.27] \text { ) }\end{array}$ & 6 \\
\hline
\end{tabular}

\begin{tabular}{|c|c|c|c|}
\hline $\begin{array}{l}\text { Controls for } \\
\text { other important } \\
\text { covariates }\end{array}$ & Confounding factors & Main results & $\begin{array}{l}\text { Total NOS } \\
\text { score }\end{array}$ \\
\hline No & $\begin{array}{l}\text { Gestational age at the interview, maternal } \\
\text { age, prior gynecologic surgery, member of } \\
\text { Jewish religion, SAB in last pregnancy }\end{array}$ & $\begin{array}{l}\text { Women consuming } \geq 151 \mathrm{mg} \text { caffeine/day had an increased } \\
\text { risk of } S A B \\
\text { RR: I.73* }\end{array}$ & 6 \\
\hline No & Age & $\begin{array}{l}\text { Women with a coffee intake of }>3 \text { cups/day had an } \\
\text { increased risk of miscarriage } \\
\text { RR: I.56 }(95 \% \mathrm{Cl} \text { I.06; } 2.30)\end{array}$ & 5 \\
\hline No & Age & $\begin{array}{l}\text { No increased risk of early pregnancy loss with caffeine } \\
\text { consumption }>3,500 \mathrm{mg} / \mathrm{month} \\
\text { RR: } 2.40(95 \% \mathrm{Cl} 0.80 ; 7.00)\end{array}$ & 6 \\
\hline Yes & $\begin{array}{l}\text { Age, race marital status, insurance coverage, } \\
\text { cigarette and alcohol consumption, previous } \\
\text { spontaneous abortions and previous } \\
\text { therapeutic abortions }\end{array}$ & $\begin{array}{l}\text { No increased risk of SAB with total caffeine intake } \\
>300 \mathrm{mg} / \text { day } \\
\text { OR: I. } 22(95 \% \mathrm{CI} 0.80 ; 1.87) \\
\text { Among heavy users, nausea modified the risk of SAB } \\
\text { OR: } 2.10(95 \% \mathrm{CI} \text { I.20; } 3.70)\end{array}$ & 7 \\
\hline No & $\begin{array}{l}\text { Adjusted for maternal age in the article (not } \\
\text { able to include this in the meta-analysis, } \\
\text { using crude estimates) }\end{array}$ & $\begin{array}{l}\text { Women with a perifertilization caffeine intake of } \\
28-124 \mathrm{mg} / \text { day had a decreased risk of SAB OR: } 0.66 \text { ( } 95 \% \\
\mathrm{CI} 0.52 ; 0.83) \\
\text { Women with a caffeine intake of } 225-1,308 \mathrm{mg} / \text { day } \\
\text { during pregnancy had an increased risk of SAB OR: I.7I } \\
(95 \% \mathrm{CI} I .29 ; 2.26)\end{array}$ & 6 \\
\hline No & $\begin{array}{l}\text { Age (adjustment for alcohol was not done in } \\
\text { the presented analysis) }\end{array}$ & $\begin{array}{l}\text { No significant increased risk of SAB with coffee intake } \\
\text { OR: I. } 40(95 \% \mathrm{Cl} 0.70 ; 2.60)\end{array}$ & 6 \\
\hline Yes & $\begin{array}{l}\text { Maternal age (at pregnancy outcome), } \\
\text { pregnancy history, educational level, ethnic } \\
\text { group, employment during pregnancy, } \\
\text { cigarettes, alcohol }\end{array}$ & $\begin{array}{l}\text { Women with a coffee intake of } 5-9 \text { cups/day had an } \\
\text { increased risk of SAB } \\
\text { OR: I.I7 ( } 95 \% \text { CI I.03; I.32) }\end{array}$ & 6 \\
\hline Yes & $\begin{array}{l}\text { Period of pregnancy, age, educational level, } \\
\text { smoking and alcohol use during pregnancy, } \\
\text { uterine abnormality, and work schedule }\end{array}$ & $\begin{array}{l}\text { Women with a pre-pregnancy caffeine intake }>321 \mathrm{mg} / \text { day } \\
\text { had an increased risk of SAB } \\
\text { OR: } 1.85(95 \% \mathrm{Cl} \text { I.I8; } 2.89) \\
\text { Women with a caffeine intake of }>321 \mathrm{mg} / \text { day during } \\
\text { pregnancy had an increased risk of SAB OR: } 2.62 \\
(95 \% \mathrm{Cl} \text { I.38; } 5.01)\end{array}$ & 7 \\
\hline
\end{tabular}


Table 2 (Continued)

\begin{tabular}{|c|c|c|c|c|c|}
\hline Author and year & Country & Design & Study groups included in the analyses & $\begin{array}{l}\text { Source of } \\
\text { caffeine }\end{array}$ & $\begin{array}{l}\text { Controls for } \\
\text { maternal age }\end{array}$ \\
\hline Mills et al, $1993^{62}$ & US & Cohort & $\begin{array}{l}\mathrm{N}=423 \text { American pregnancy planners ( } 24 \% \\
\text { enrolled no later than } 2 \text { I days after conception) } \\
\text { Cases: } 59\end{array}$ & Multiple & Yes \\
\hline $\begin{array}{l}\text { Dominguez-Rojas } \\
\text { et al, } 1994^{54}\end{array}$ & Spain & Cohort & $\begin{array}{l}\mathrm{N}=691 \text { Spanish women who were primipara } \\
\text { Cases: } 169\end{array}$ & Coffee & Yes \\
\hline $\begin{array}{l}\text { Lubna and Al-Ansary, } \\
1994^{37}\end{array}$ & Saudi Arabia & $\begin{array}{l}\text { Case- } \\
\text { control }\end{array}$ & $\begin{array}{l}\mathrm{N}=452 \\
\text { Cases: } 226 \text { Saudi women hospitalized for SAB } \\
\text { within the first } 4 \text { weeks of gestation } \\
\text { Controls: } 226 \text { women admitted for normal } \\
\text { delivery }\end{array}$ & Multiple & No \\
\hline Dlugosz et al, $1996^{55}$ & US & Cohort & $\begin{array}{l}\mathrm{N}=2,849 \text { pregnant American women recruited } \\
\text { from private clinics } \\
\text { Cases: } 135\end{array}$ & $\begin{array}{l}\text { Multiple/ } \\
\text { coffee }\end{array}$ & Yes \\
\hline $\begin{array}{l}\text { Zhang and Bracken, } \\
1996^{67}\end{array}$ & US & Cohort & $\begin{array}{l}\mathrm{N}=2,849 \text { pregnant American women recruited } \\
\text { from private clinics } \\
\text { Cases: I } 35\end{array}$ & Coffee & No \\
\hline Agnesi et al, $1997^{57}$ & Italy & $\begin{array}{l}\text { Case-- } \\
\text { control }\end{array}$ & $\begin{array}{l}\mathrm{N}=216 \\
\text { Cases: } 108 \text { Italian women with clinically } \\
\text { recognized SAB } \\
\text { Controls: } 108 \text { women admitted for normal } \\
\text { delivery; matched by age, year, and residence }\end{array}$ & Coffee & Yes \\
\hline Fenster et al, $1997^{52}$ & US & Cohort & $\begin{array}{l}\mathrm{N}=5,142 \text { pregnant American women enrolled } \\
\text { from three private facilities } \\
\text { Cases: } 498\end{array}$ & Multiple & Yes \\
\hline Parazzini et al, $1998^{63}$ & Italy & $\begin{array}{l}\text { Case- } \\
\text { control }\end{array}$ & $\begin{array}{l}\mathrm{N}=2,325 \\
\text { Cases: } 782 \text { Italian women admitted for SAB within } \\
\text { the first I } 2 \text { weeks of gestation } \\
\text { Controls: I,543 women who gave birth at term } \\
\text { (>37 weeks of gestation) }\end{array}$ & Coffee & Yes \\
\hline $\begin{array}{l}\text { Cnattingius et al, } \\
2000^{32}\end{array}$ & Sweden & $\begin{array}{l}\text { Case-- } \\
\text { control }\end{array}$ & $\begin{array}{l}\mathrm{N}=1,515 \\
\text { Cases: } 562 \text { Swedish women admitted with SAB } \\
\text { within 6-12 weeks of gestation } \\
\text { Controls: } 953 \text { pregnant women, frequency- } \\
\text { matched on weeks of gestation and area of } \\
\text { residence }\end{array}$ & Multiple & Yes \\
\hline Wen et al, 200I ${ }^{68}$ & US & Cohort & $\begin{array}{l}\mathrm{N}=550 \text { American women planning to become } \\
\text { pregnant and who had not been trying for more } \\
\text { than } 3 \text { months at enrolment } \\
\text { Cases: } 70\end{array}$ & Multiple & No \\
\hline
\end{tabular}




\begin{tabular}{|c|c|c|c|}
\hline $\begin{array}{l}\text { Controls for } \\
\text { other important } \\
\text { covariates }\end{array}$ & Confounding factors & Main results & $\begin{array}{l}\text { Total NOS } \\
\text { score }\end{array}$ \\
\hline Yes & $\begin{array}{l}\text { Smoking, maternal age, parity, prior SAB, } \\
\text { alcohol use, maternal education, and income }\end{array}$ & $\begin{array}{l}\text { No increased risk of SAB with caffeine intake } \\
\text { OR: I.I5 (95\% Cl 0.89; I.49) }\end{array}$ & 8 \\
\hline No & $\begin{array}{l}\text { Age, previous SAB, menarcheal age, and } \\
\text { marital status }\end{array}$ & $\begin{array}{l}\text { Women with a caffeine intake of I4I-280, } 28 I-420 \text {, and } \\
>420 \mathrm{mg} / \text { day had an increased risk of SAB } \\
\text { OR: } 2.20 \text { ( } 95 \% \mathrm{CI} \text { I.22; 3.96), OR: } 4.8 \mathrm{I}(95 \% \mathrm{Cl} 2.28 \text {; } \\
\text { I0.I4), and OR: I5.43 (95\% CI 7.34; } 32.43)\end{array}$ & 5 \\
\hline No & No & $\begin{array}{l}\text { Women with a caffeine intake of }>150 \mathrm{mg} / \text { day had an } \\
\text { increased risk of SAB RR: } 1.90(95 \% \mathrm{Cl} 1.20 ; 3.00)\end{array}$ & 5 \\
\hline Yes & $\begin{array}{l}\text { Maternal age, gestational age at the } \\
\text { interview, cigarette smoking, and alcoholic } \\
\text { beverage consumption } \\
\text { (also adjusted for tea and soda intake when } \\
\text { reporting results on coffee intake) }\end{array}$ & $\begin{array}{l}\text { Women with a coffee intake of }>3 \text { cups/day had an } \\
\text { increased risk of SAB } \\
\text { OR: } 2.63(95 \% \mathrm{CI} \text { I.29; } 5.34)\end{array}$ & 8 \\
\hline No & No & $\begin{array}{l}\text { Women consuming } \geq 3 \text { cups of coffee/day during the first } \\
\text { month of pregnancy had an increased risk of SAB } \\
\text { RR: } 2.45(95 \% \mathrm{Cl} \text { I.38; } 4.35)\end{array}$ & 3 \\
\hline Yes & $\begin{array}{l}\text { Gravidity, previous abortions, level of } \\
\text { education, smoking habits, consumption } \\
\text { of alcohol, medicines and marital status, } \\
\text { solvent exposure }\end{array}$ & $\begin{array}{l}\text { Women with a coffee intake had an increased risk of SAB } \\
\text { RR: } 1.36(95 \% \mathrm{Cl} \text { I.06; I.76) }\end{array}$ & 6 \\
\hline Yes & $\begin{array}{l}\text { Maternal age, pregnancy history, cigarette } \\
\text { smoking, alcohol consumption, employment, } \\
\text { race, gestational age at interview, marital } \\
\text { and socioeconomic status (also adjusted for } \\
\text { tea, soda, and decaffeinated coffee when } \\
\text { analyzing caffeinated coffee) }\end{array}$ & $\begin{array}{l}\text { No increased risk of SAB with a prepregnancy intake of } \\
\text { total caffeine }>300 \mathrm{mg} / \text { day before pregnancy } \\
\text { OR: I.25 }(95 \% \mathrm{Cl} 0.90 ; \text { I.73) or coffee }>3 \text { cups/day } \\
\text { OR: I.I3 }(95 \% \mathrm{Cl} 080 ; \text { I.60) } \\
\text { During first trimester, there were no increased risk of SAB } \\
\text { with total caffeine intake }>300 \mathrm{mg} / \text { day } \\
\text { OR: } 1.29(95 \% \mathrm{Cl} 0.80 ; 2.06) \\
\text { or coffee intake }>3 \text { cups/day } \\
\text { OR: } 0.84(95 \% \mathrm{Cl} 0.4 \mathrm{I} ; \mathrm{I} .68)\end{array}$ & 7 \\
\hline Yes & $\begin{array}{l}\text { Age, education, previous live births, and } \\
\text { SAB, maternal alcohol consumption and } \\
\text { smoking in the first trimester, nausea } \\
\text { intensity in the first trimester of pregnancy }\end{array}$ & $\begin{array}{l}\text { Women with a coffee consumption of } 2-3 \text { and } \geq 4 \text { cups/day } \\
\text { before conception had an increased risk of SAB } \\
\text { OR: I.34 ( } 95 \% \mathrm{Cl} \text { I.04; I.73) and OR: I. } 47(95 \% \mathrm{Cl} \text { I.07; } 2.02) \\
\text { Women with a coffee consumption of } 2-3 \text { and } \geq 4 \text { cups/day } \\
\text { during the first trimester had an increased risk of SAB } \\
\text { OR: I.75 }(95 \% \mathrm{Cl} \text { I.23; } 2.29) \text { and OR: } 3.98(95 \% \mathrm{Cl} 2.55 ; 6.2 \mathrm{I})\end{array}$ & 8 \\
\hline Yes & $\begin{array}{l}\text { Smoking status, age, number of previous } \\
\text { pregnancies, history of SAB, consumption of } \\
\text { alcohol during pregnancy (yes/no), presence } \\
\text { or absence of nausea, vomiting, and fatigue }\end{array}$ & $\begin{array}{l}\text { No increased risk of early } S A B \text { with total caffeine intake } \\
\geq 500 \mathrm{mg} / \text { day during pregnancy } \\
\text { OR: I.40 ( } 95 \% \mathrm{Cl} 0.90 ; 2.20) \\
\text { When stratifying on smoking status, nonsmokers with a } \\
\text { caffeine intake } \geq 500 \mathrm{mg} / \text { day had an increased risk of SAB } \\
\text { OR: } 2.20 \text { ( } 95 \% \mathrm{Cl} 1.30 ; 3.80) \\
\text { No association was found among smokers }\end{array}$ & 8 \\
\hline No & No & $\begin{array}{l}\text { No increased risk for } S A B \text { with total caffeine intake of } \\
\geq 300 \mathrm{mg} / \text { day before pregnancy } \\
\text { RR: I.IO ( } 95 \% \mathrm{Cl} 0.50 ; 2.40) \\
\text { A tendency of increased risk for SAB with total caffeine } \\
\text { intake } \geq 300 \mathrm{mg} / \text { day during first trimester } \\
\text { RR: } 2.50(95 \% \mathrm{Cl} \text { I.00; } 6.40)\end{array}$ & 3 \\
\hline
\end{tabular}


Table 2 (Continued)

\begin{tabular}{|c|c|c|c|c|c|}
\hline Author and year & Country & Design & Study groups included in the analyses & $\begin{array}{l}\text { Source of } \\
\text { caffeine }\end{array}$ & $\begin{array}{l}\text { Controls for } \\
\text { maternal age }\end{array}$ \\
\hline Giannelli et al, $2003^{58}$ & UK & $\begin{array}{l}\text { Case- } \\
\text { control }\end{array}$ & $\begin{array}{l}\mathrm{N}=469 \\
\text { Cases: } 159 \text { nulliparous English women with a } \\
\text { clinically recognized miscarriage } \\
\text { Controls: } 310 \text { nulliparous pregnant women, } \\
\text { without a SAB in Ist and 2nd trimesters }\end{array}$ & Multiple & Yes \\
\hline Rasch, $2003^{77}$ & Denmark & $\begin{array}{l}\text { Case- } \\
\text { control }\end{array}$ & $\begin{array}{l}\mathrm{N}=1,459 \\
\text { Cases: } 320 \text { Danish women with an SAB in } \\
\text { gestational week 6-16 } \\
\text { Controls: I, I } 39 \text { pregnant women with a live fetus } \\
\text { at gestational week 6-16 }\end{array}$ & Multiple & Yes \\
\hline Tolstrup et al, $2003^{69}$ & Denmark & $\begin{array}{l}\text { Case- } \\
\text { control }\end{array}$ & $\begin{array}{l}\mathrm{N}=1,712 \\
\text { Cases: } 303 \text { Danish women who reported an } \mathrm{SAB} \\
\text { or registered with a diagnosis of } \mathrm{SAB} \\
\text { Controls: } 1,409 \text { women reporting giving birth or } \\
\text { being pregnant for }>28 \text { weeks }\end{array}$ & Multiple & Yes \\
\hline Khoury et al, $2004^{35}$ & US & Cohort & $\begin{array}{l}\mathrm{N}=191 \text { pregnant American women with type I } \\
\text { diabetes planning a pregnancy or enrolled after } \\
\text { conception } \\
\text { Cases: } 23\end{array}$ & Multiple & Yes \\
\hline Bech et al, $2005^{21}$ & Denmark & Cohort & $\begin{array}{l}\mathrm{N}=86,282 \text { pregnant Danish women recruited by } \\
\text { their GP } \\
\text { Cases: } 616\end{array}$ & Coffee & Yes \\
\hline Sata et al, $2005^{70}$ & Japan & $\begin{array}{l}\text { Case- } \\
\text { control }\end{array}$ & $\begin{array}{l}\mathrm{N}=205 \\
\text { Cases: } 58 \text { Japanese women with a history of } \\
\text { recurrent pregnancy loss } \\
\text { Controls: } 147 \text { fertile women whose pregnancies } \\
\text { ended in live births }\end{array}$ & Multiple & Yes \\
\hline George et al, $2006^{59}$ & Sweden & $\begin{array}{l}\text { Case- } \\
\text { control }\end{array}$ & $\begin{array}{l}\mathrm{N}=69 \mathrm{I} \\
\text { Cases: } 108 \text { Swedish women admitted with two or } \\
\text { more consecutive miscarriages } \\
\text { Controls: } 583 \text { pregnant women with at least two } \\
\text { pregnancies, frequency-matched on weeks of } \\
\text { gestation and area of residence }\end{array}$ & Multiple & Yes \\
\hline $\begin{array}{l}\text { Maconochie et al, } \\
2007^{64}\end{array}$ & UK & $\begin{array}{l}\text { Case- } \\
\text { control }\end{array}$ & $\begin{array}{l}\mathrm{N}=6,167 \\
\text { Cases: } 546 \text { English women whose most recent } \\
\text { pregnancy had ended in first trimester miscarriage } \\
(<13 \text { weeks of gestation) or who had a miscarriage } \\
\text { since } 1995 \\
\text { Controls: } \mathrm{N}=5,62 \mathrm{I} \text { women whose most recent } \\
\text { pregnancy progressed beyond } 13 \text { weeks of } \\
\text { gestation }\end{array}$ & Multiple & Yes \\
\hline Savitz et al, $2008^{71}$ & US & Cohort & $\begin{array}{l}\mathrm{N}=2,370 \text { pregnant American women enrolled } \\
\text { before } 12 \text { weeks of gestation and who had not } \\
\text { been trying to conceive for }>6 \text { months } \\
\text { Cases: } 258\end{array}$ & $\begin{array}{l}\text { Multiple/ } \\
\text { coffee }\end{array}$ & Yes \\
\hline
\end{tabular}




\begin{tabular}{|c|c|c|c|}
\hline $\begin{array}{l}\text { Controls for } \\
\text { other important } \\
\text { covariates }\end{array}$ & Confounding factors & Main results & $\begin{array}{l}\text { Total NOS } \\
\text { score }\end{array}$ \\
\hline No & $\begin{array}{l}\text { Maternal age, nausea in pregnancy, and } \\
\text { gestational age }\end{array}$ & 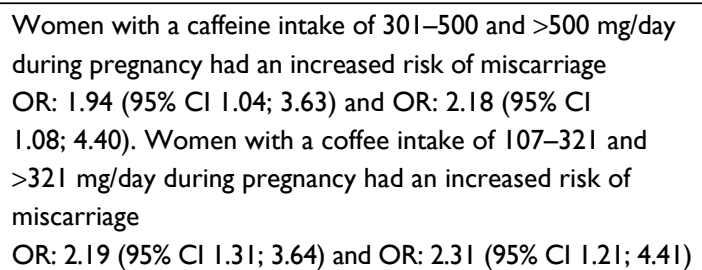 & 6 \\
\hline Yes & $\begin{array}{l}\text { Age, parity, occupation, cigarette smoking, } \\
\text { and alcohol consumption }\end{array}$ & $\begin{array}{l}\text { Women with a caffeine intake of } \geq 375 \mathrm{mg} / \text { day had an } \\
\text { increased risk of } \mathrm{SAB} \\
\text { OR: } 2.21 \text { ( } 95 \% \mathrm{Cl} \text { I.53; } 3.18)\end{array}$ & 8 \\
\hline Yes & $\begin{array}{l}\text { Maternal age, marital status, smoking, and } \\
\text { alcohol intake }\end{array}$ & $\begin{array}{l}\text { A tendency of increased risk for SAB with total caffeine } \\
\text { intake of }>900 \mathrm{mg} / \text { day } \\
\text { OR: } 1.72(95 \% \mathrm{Cl} \text { I.00; } 2.96) \\
\text { No interaction between caffeine and smoking }\end{array}$ & 6 \\
\hline No & $\begin{array}{l}\text { Maternal age, years since diagnosis of } \\
\text { diabetes, previous SAB, nephropathy and } \\
\text { retinopathy, glycemic control, and cigarette } \\
\text { smoking }\end{array}$ & $\begin{array}{l}\text { Women who consumed caffeine early in the first trimester } \\
\text { had an increased risk of SAB } 4.50(95 \% \mathrm{Cl} 1.20 ; 16.80) \\
\text { compared to women not consuming caffeine }\end{array}$ & 4 \\
\hline Yes & $\begin{array}{l}\text { Age, parity, smoking, prepregnancy } \\
\text { BMI, alcohol consumption, and socio- } \\
\text { occupational status }\end{array}$ & $\begin{array}{l}\text { Women with a coffee intake of } \geq 8 \text { cups/day had an } \\
\text { increased risk of fetal death (gestation length }<140 \text { days) } \\
\text { HR: } 1.48(95 \% \mathrm{Cl} 1.01 ; 2.17)\end{array}$ & 7 \\
\hline No & Age and smoking status during pregnancy & $\begin{array}{l}\text { No statistical significant increased risk of RPL with a } \\
\text { caffeine intake of } \geq 300 \mathrm{mg} / \text { day during pregnancy when } \\
\text { compared to women with a caffeine intake of } 0-99 \mathrm{mg} / \text { day } \\
\text { OR: I.82 ( } 95 \% \mathrm{Cl} 0.72 ; 4.58) \\
\text { Women with homozygous CYPIA2*IF alleles had an } \\
\text { increased risk of RPL with a caffeine intake of } \geq 300 \mathrm{mg} / \text { day } \\
\text { OR: } 5.23 \text { ( } 95 \% \mathrm{Cl} \mathrm{I.05;25.90)}\end{array}$ & 6 \\
\hline Yes & $\begin{array}{l}\text { Maternal age, obstetric history, induced } \\
\text { abortions, myoma, time to concieve, marital } \\
\text { status, smoking, alcohol intake during } \\
\text { pregnancy, amd plasma folate levels }\end{array}$ & $\begin{array}{l}\text { No statistical significant increased risk of RPL with a } \\
\text { caffeine intake of } \geq 300 \mathrm{mg} / \text { day during pregnancy } \\
\text { OR: } 1.80(95 \% \mathrm{Cl} 0.80 ; 2.90) \\
\text { Nonsmokers had an increased risk of RPL with a caffeine } \\
\text { intake of } \geq 300 \mathrm{mg} / \text { day } \\
\text { OR: } 2.70(95 \% \mathrm{Cl} \mathrm{I.I0;6.20)}\end{array}$ & 8 \\
\hline No & $\begin{array}{l}\text { Year of conception, maternal age at } \\
\text { conception, pregnancy order, history of } \\
\text { miscarriage, history of live birth } \\
\text { (further adjusted for nausea in one analysis) }\end{array}$ & 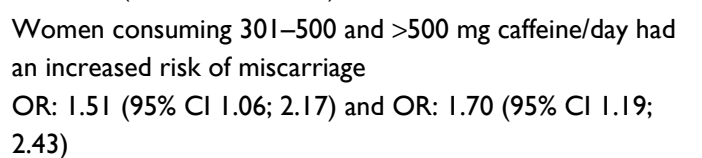 & 5 \\
\hline Yes & $\begin{array}{l}\text { Maternal age, race/etnicity, maternal } \\
\text { education, marital status, alcohol use, } \\
\text { vitamin use and symptoms of nausea, and } \\
\text { vomiting during early pregnancy }\end{array}$ & $\begin{array}{l}\text { No increased risk of SAB with a prepregnancy coffee } \\
\text { intake of }>696 \mathrm{mg} / \text { day OR: } 0.90(95 \% \mathrm{Cl} 0.50 ; \mathrm{I} .50) \text { or } \\
\text { total caffeine intake of }>5 \mathrm{I} 3.2 \mathrm{mg} / \text { day } \\
\text { OR: } 0.80(95 \% \mathrm{Cl} 0.50 ; \mathrm{I} .20) \\
\text { No increased risk of SAB with a coffee intake of } \\
>372.9 \mathrm{mg} / \text { day at the time of the telephone interview } \\
\text { OR: } 0.70(95 \% \mathrm{Cl} 0.30 ; \text { I.80) or total caffeine intake of } \\
>273.2 \mathrm{mg} / \text { day } \\
\text { OR: } 1.30(95 \% \mathrm{Cl} 0.90 ; \text { I. } 19)\end{array}$ & 8 \\
\hline
\end{tabular}


Table 2 (Continued)

\begin{tabular}{|c|c|c|c|c|c|}
\hline Author and year & Country & Design & Study groups included in the analyses & $\begin{array}{l}\text { Source of } \\
\text { caffeine }\end{array}$ & $\begin{array}{l}\text { Controls for } \\
\text { maternal age }\end{array}$ \\
\hline Weng et al, $2008^{72}$ & US & Cohort & $\begin{array}{l}\mathrm{N}=1,063 \text { pregnant American women enrolled } \\
\text { before } 14 \text { weeks of gestation } \\
\text { Cases: } 172\end{array}$ & $\begin{array}{l}\text { Multiple/ } \\
\text { coffee }\end{array}$ & Yes \\
\hline Agnesi et al, $2010^{56}$ & Italy & $\begin{array}{l}\text { Case- } \\
\text { control }\end{array}$ & $\begin{array}{l}\mathrm{N}=245 \\
\text { Cases: I } 23 \text { Italian women with a clinically } \\
\text { recognized SAB } \\
\text { Controls: I } 22 \text { women admitted for normal } \\
\text { delivery, matched by age, year, and residence }\end{array}$ & Coffee & Yes \\
\hline $\begin{array}{l}\text { Greenwood et al, } \\
2010^{65}\end{array}$ & UK & Cohort & $\begin{array}{l}\mathrm{N}=2,482 \text { pregnant English women } \\
\text { Cases: } 25\end{array}$ & $\begin{array}{l}\text { Multiple/ } \\
\text { coffee }\end{array}$ & Yes \\
\hline Pollack et al, $2010^{29}$ & US & Cohort & $\begin{array}{l}\mathrm{N}=67 \text { American women planning a pregnancy } \\
\text { within the next } 5 \text { years and who became pregnant } \\
\text { Cases: } 14\end{array}$ & Coffee & Yes \\
\hline $\begin{array}{l}\text { Stefanidou et al, } \\
201 \mathrm{I}^{33}\end{array}$ & Italy & $\begin{array}{l}\text { Case- } \\
\text { control }\end{array}$ & $\begin{array}{l}\mathrm{N}=312 \\
\text { Cases: } 52 \text { Italian women with sine causa recurrent } \\
\text { miscarriages } \\
\text { Controls: } 260 \text { women with healthy pregnancies }\end{array}$ & Multiple & Yes \\
\hline Hanh et al, $2015^{73}$ & Denmark & Cohort & $\begin{array}{l}\mathrm{N}=5,132 \text { Danish women planning a pregnancy, } \\
\text { I8-40 years } \\
\text { Cases: } 732\end{array}$ & $\begin{array}{l}\text { Multiple/ } \\
\text { Coffee }\end{array}$ & Yes \\
\hline
\end{tabular}

Abbreviations: RPL, recurrent pregnancy loss; NOS, Newcastle-Ottawa scale; MF, magnetic fields; TTP, time to pregnancy; SAB, spontaneous abortion; BMI, body mass index; $R R$, relative risk; METS, estimated total metabolic equivalents; OR, odds ratio; HR, hazards ratio.

Table 3 Characteristics and results of publications on coffee/caffeine and MAR treatment

\begin{tabular}{|c|c|c|c|c|c|}
\hline Author and year & Country & Design & $\begin{array}{l}\text { Study groups } \\
\text { included in the analyses }\end{array}$ & $\begin{array}{l}\text { Source of } \\
\text { caffeine }\end{array}$ & $\begin{array}{l}\text { Controls for } \\
\text { maternal age }\end{array}$ \\
\hline $\begin{array}{l}\text { Klonoff-Cohen et al, } \\
2002^{74}\end{array}$ & US & Cohort & $\begin{array}{l}\mathrm{N}=192 \text { American women with primary or } \\
\text { secondary infertility undergoing their first } \\
\text { fresh, non-donor IVF, or GIFT cycle } \\
\text { Cases: } 40\end{array}$ & Multiple & Yes \\
\hline Al-Saleh et al, $2010^{75}$ & Saudi Arabia & Cohort & $\begin{array}{l}\mathrm{N}=474 \text { Saudi Arabian women undergoing } \\
\text { their first IVF treatment } \\
\text { Cases: I } 84\end{array}$ & Multiple/coffee & Yes \\
\hline Choi et al, 201 I76 & US & Cohort & $\begin{array}{l}\mathrm{N}=2,474 \text { American women undergoing } \\
\text { IVF treatment at three fertility clinics in } \\
\text { Boston and contributing } 4,7 \text { I } 6 \text { IVF cycles } \\
\text { Cases: I, I } 47\end{array}$ & Multiple & Yes \\
\hline
\end{tabular}

Abbreviations: GIFT, gamete intra-fallopian transfer; NOS, Newcastle-Ottawa scale; IVF, in vitro fertilization; SAB, spontaneous abortion; BMI, body mass index; OR, odds ratio. 


\begin{tabular}{|c|c|c|c|}
\hline $\begin{array}{l}\text { Controls for } \\
\text { other important } \\
\text { covariates }\end{array}$ & Confounding factors & Main results & $\begin{array}{l}\text { Total NOS } \\
\text { score }\end{array}$ \\
\hline Yes & $\begin{array}{l}\text { Maternal age, race, education, household } \\
\text { income, marital status, previous } \\
\text { miscarriages, smoking, alcohol consumption, } \\
\text { jacuzzi use, MF exposure, and nausea and } \\
\text { vomiting }\end{array}$ & $\begin{array}{l}\text { Women consuming }>200 \mathrm{mg} \text { caffeine/day had an increased } \\
\text { risk of miscarriage } \\
\text { HR: } 2.23(1.34 ; 3.69)\end{array}$ & 6 \\
\hline No & No & $\begin{array}{l}\text { A tendency toward an increased risk of SAB with a coffee } \\
\text { intake of } 4 \text { cups/day } \\
\text { OR: } 4.48(0.44 ; 2.23)\end{array}$ & 5 \\
\hline Yes & $\begin{array}{l}\text { Maternal age, parity, amount smoked } \\
\text { (cotinine concentration) and alcohol intake } \\
\text { (no change in results by further adjusting } \\
\text { for nausea) }\end{array}$ & $\begin{array}{l}\text { Women with a caffeine intake of }>300 \mathrm{mg} / \text { day had an } \\
\text { increased risk of late miscarriage } \\
\text { OR: } 5.10(1.60 ; 16.40)\end{array}$ & 8 \\
\hline Yes & $\begin{array}{l}\text { Age, average alcohol consumption, and } \\
\text { cigarette consumption per standardized } \\
\text { 28-day cycle }\end{array}$ & $\begin{array}{l}\text { Women with an intake of caffeine did not have an } \\
\text { increased risk of miscarriage } \\
\text { RR: } 0.98(0.96 ; 0.99)\end{array}$ & 6 \\
\hline Yes & $\begin{array}{l}\text { Maternal age at conception, cigarette } \\
\text { smoking, alcohol consumption, maternal } \\
\text { education nausea/vomiting }\end{array}$ & $\begin{array}{l}\text { For each } 100 \mathrm{mg} \text { increase in caffeine intake per day the } \\
\text { risk of sine causa recurrent miscarriage increased } \\
\text { OR: } 2.72(2.71 ; 2.73)\end{array}$ & 7 \\
\hline Yes & $\begin{array}{l}\text { Maternal age, physical activity, parity, BMI, } \\
\text { vocational training/education, smoking, prior } \\
\text { SAB, alcohol (preconception estimate) }\end{array}$ & $\begin{array}{l}\text { Preconception caffeine consumption was not appreciably } \\
\text { associated with SAB overall } \\
\text { Early pregnancy caffeine consumption was associated with } \\
\text { a slightly increased risk of SAB HR: } 1.48(95 \% \mathrm{Cl} \text { I.03; } \\
2.13) \text { when comparing a caffeine intake of } 200-299 \mathrm{mg} / \text { day } \\
\text { with } \leq 100 \mathrm{mg} / \text { day }\end{array}$ & 7 \\
\hline
\end{tabular}

\begin{tabular}{|c|c|c|c|}
\hline $\begin{array}{l}\text { Controls for } \\
\text { other important } \\
\text { covariates }\end{array}$ & Confounding factors & Main results & $\begin{array}{l}\text { Total NOS } \\
\text { score }\end{array}$ \\
\hline Yes & $\begin{array}{l}\text { Smoking, alcohol use, age, race, education, } \\
\text { parity, type of infertility, type of procedure, } \\
\text { number of attempts, number of embryos } \\
\text { transferred }\end{array}$ & $\begin{array}{l}\text { Women with a caffeine intake of }>2-50 \mathrm{mg} / \text { day during the } \\
\text { week of the initial clinical visit had an increased risk of SAB } \\
\text { OR: } 9.50 \text { ( } 95 \% \mathrm{Cl} \text { I.40; } 62.00) \\
\text { Women with a caffeine intake of }>2-50 \text { or }>50 \mathrm{mg} / \text { day during } \\
\text { the week of the initial clinical visit had an increased risk of not } \\
\text { achieving a live birth OR: } 2.90 \text { ( } 95 \% \mathrm{Cl} \text { I.10; } 7.50) \text { and OR: } \\
3.80 \text { ( } 95 \% \mathrm{Cl} \text { I.40; } 10.70) \text {, respectively) }\end{array}$ & 7 \\
\hline Yes & $\begin{array}{l}\text { Women's age, BMI, cause of infertility, } \\
\text { family income, smoking status, use of } \\
\text { herbal treatment, and health status }\end{array}$ & $\begin{array}{l}\text { No association between the chance of achieving a pregnancy } \\
\text { and a coffee intake of }>10 \text { cups/day OR: } 0.77(95 \% \mathrm{Cl} 0.22 ; 2.74) \\
\text { No association between the chance of achieving a pregnancy and } \\
\text { serum-caffeine OR: } 0.93(95 \% \mathrm{Cl} 0.65 ; 1.33)\end{array}$ & 7 \\
\hline Yes & $\begin{array}{l}\text { Female age, BMI, clinic site, study } \\
\text { enrollment period, female tobacco use, } \\
\text { female alcohol use, primary infertility } \\
\text { diagnosis }\end{array}$ & $\begin{array}{l}\text { No association between female caffeine consumption and the } \\
\text { live birth rate } \\
\text { Women with a caffeine intake of I-I I } 4 \mathrm{mg} / \text { day or }>200 \mathrm{mg} / \text { day } \\
\text { had no decreased risk of achieving a live birth OR: I.00 } \\
(95 \% \mathrm{Cl} 0.83 ; \text { I.2I) and OR: I.07 ( } 95 \% \mathrm{Cl} 0.85 ; \mathrm{I} .34) \text {, respectively }\end{array}$ & 7 \\
\hline
\end{tabular}


$100 \mathrm{mg}$ caffeine/day, 1.37 (95\% CI: 1.19; 1.57) for $300 \mathrm{mg}$ caffeine/day, and 2.32 (95\% CI: 1.62; 3.31) for $600 \mathrm{mg}$ caffeine/day. There was evidence of significant between-study heterogeneity $\left(I^{2}=73,7 \% ; p<0.0001\right)$, and Egger's regression test provided evidence of substantial publication bias $(p<0.0001$; Table 2).

We stratified the SAB analyses on type of consumption, adjustment status, study quality, and study design. The analyses revealed that the associations were similar between studies reporting caffeine and coffee consumption ( $p$ for heterogeneity $=0.87$ ). Also, the associations were similar across strata according to adjustment status (ie, studies with adjusted results compared to unadjusted results) ( $p$ for heterogeneity $=0.44$ ) and according to study quality (ie, comparing studies with NOS $\geq 7$ with those of NOS $<7$ ) ( $p$ for heterogeneity=0.22). We found weak evidence of a difference by study design with the associations being more pronounced in cohort studies than in case-control studies ( $p$ for heterogeneity $=0.04)$. In a sensitivity analysis, changing the conversion factor of caffeine contained in one cup of coffee from 100 $\mathrm{mg}$ to $150 \mathrm{mg}$ did not substantially change the results.

\section{Studies not eligible for meta-analysis}

Five other studies, not included in the meta-analysis, have investigated a possible association between coffee/caffeine consumption and SAB with consistent findings in line with our results. A Swedish cohort study including 654 women $^{31}$ reported an increased risk of $\mathrm{SAB}$ with a daily coffee consumption greater than three cups, while a case-control study among 452 SaudiArabian women ${ }^{37}$ showed an association at caffeine consumption levels $>150 \mathrm{mg} /$ day. Also, the association of an increased risk of $\mathrm{SAB}$ with caffeine consumption was found among a selected group of 191 American women with type-1 diabetes. ${ }^{35}$ In an Italian case-control study including 312 women, ${ }^{33}$ a dose-response association was reported between caffeine intake and risk of recurrent SAB. Also, Parazzini et $\mathrm{al}^{36}$ found an association with any coffee consumption among women with recurrent pregnancy loss in a case-control study including 260 women; however, results were not statistically significant.

\section{MAR treatment}

Only two cohort studies provided sufficient data on live birth rate among couples undergoing in vitro fertilization (IVF)

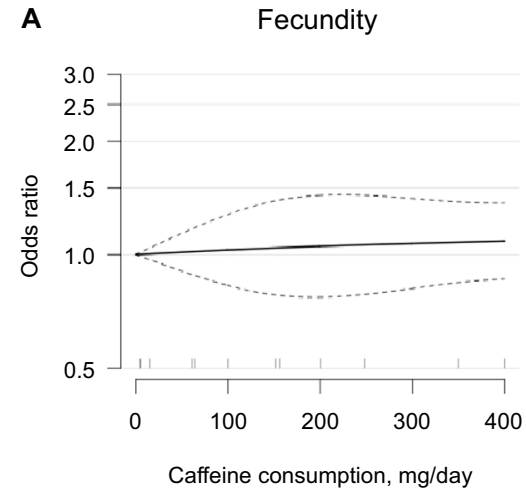

Caffeine consumption, mg/day
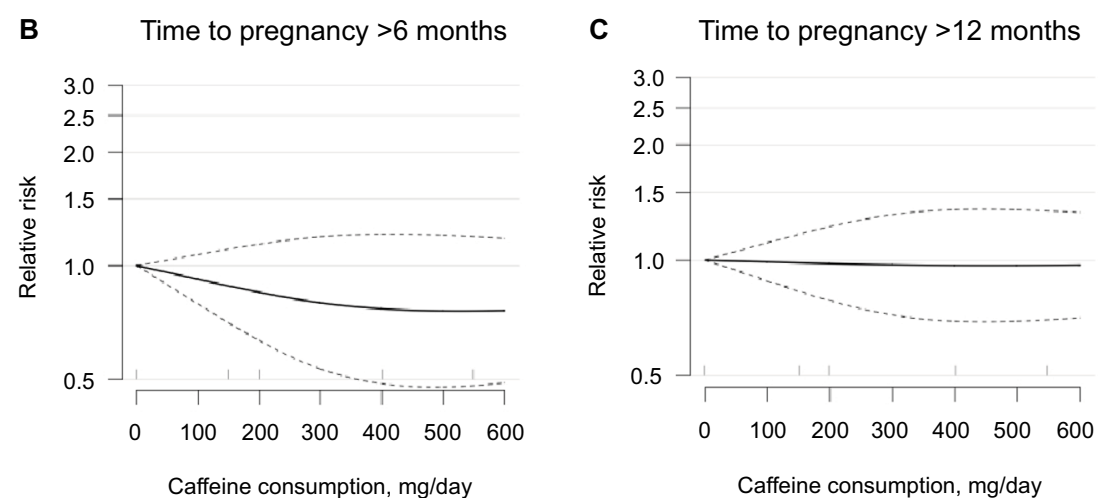
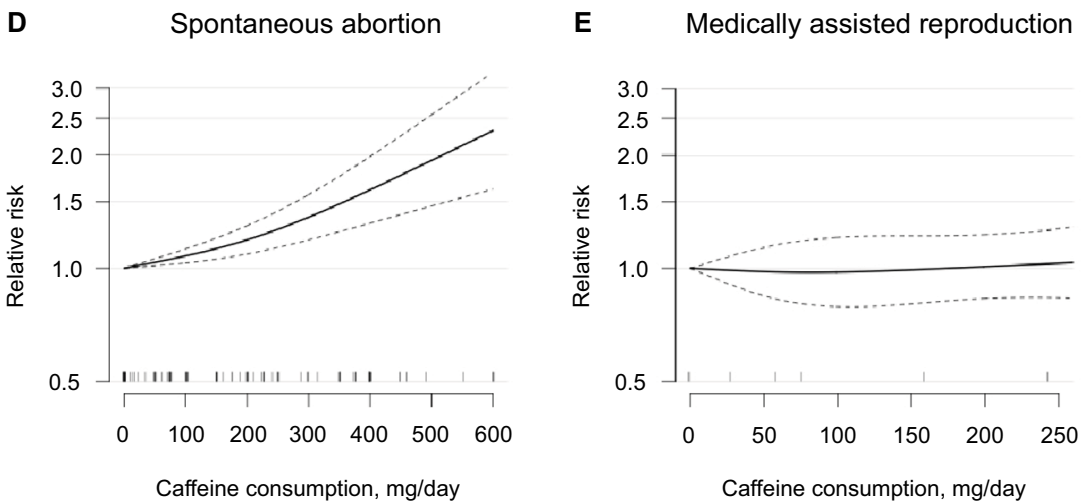

Figure 2 Dose-response association between caffeine consumption and odds ratio of fecundability (A), relative risk of time to pregnancy $>6$ months (B) and $>12$ months (C), spontaneous abortion (D), and live birth rate among couples receiving MAR treatment (E) compared to 0 mg consumption as reference. Odds ratios/relative risks are plotted on the log scale with $95 \%$ confidence intervals for the spline model. Tick marks on the x-axis represent category medians of exposure from the included studies. Abbreviations: MAR, medically assisted reproduction. 
treatment ${ }^{74,76}$ to be included in the dose-response metaanalysis. The results did not indicate an association between caffeine intake and the live birth rate among women in fertility treatment ( $p=0.94$; Figure $2 \mathrm{E}$ ). The pooled relative risks for achieving a live birth were 0.99 (95\% CI: $0.89 ; 1.10)$ for $100 \mathrm{mg}$ caffeine/day and 1.00 (95\% CI: $0.80 ; 1.24)$ for 300 $\mathrm{mg}$ caffeine/day, compared with no caffeine intake.

\section{Study not eligible for meta-analysis}

Only one study by Al-Saleh et a ${ }^{75}$ reported on the pregnancy rate among couples undergoing fertility treatment. The study showed no association between coffee/tea consumption or serum caffeine concentration and the success rate of pregnancy among 619 Saudi-Arabian women undergoing their first IVF-treatment cycle. However, the number of eggs was lower at higher levels of serum caffeine.

\section{Discussion}

This systematic review and meta-analysis showed no clear association between exposure to coffee/caffeine and natural fertility as measured by FOR or waiting TTP. However, due to the limited number of studies included, no firm conclusions can be drawn for these endpoints. Our pooled results indicate that coffee/caffeine consumption is associated with a significantly increased risk of $\mathrm{SAB}$, as expected from previous reviews and meta-analyses..$^{23,78,79}$ Further, in addition to previous studies, we intended to capture nonlinear associations using novel statistical methods but found no evidence of a "threshold effect". With regard to the potential effect of coffee or caffeine consumption on the outcomes of fertility treatment, our sparse results on MAR treatment as measured by the pregnancy and live birth rate in connection with IVF treatment did not show an association. Yet, the data available enable us to conclude little about the potential dose-response relationship.

Various hypotheses exist concerning the possible mechanisms of the potential effect of caffeine on different fecundity and pregnancy outcomes, but the eventual mechanisms are still unclear. Within the human body, caffeine acts as a nonselective adenosine antagonist $;{ }^{80}$ it increases the intracellular concentration of cyclic AMP ${ }^{81}$ and alters the blood levels of catecholamines. ${ }^{82}$ Coffee and caffeine consumption may affect the reproductive health by affecting endogenous hormone levels through changes in ovarian function or alterations in hormone metabolism: numerous studies have reported an inverse association between coffee/caffeine intake and levels of free estradiol ${ }^{83-86}$ in line with the increased levels of sex hormone-binding globulin reported by others. ${ }^{87-89}$
However, contradicting results of increased levels ${ }^{76,90}$ or no effects on estradiol levels are also reported. ${ }^{87,88,91,92}$ Caffeine and estradiol are both metabolized by the hepatic enzyme CYP1A2 ${ }^{93,94}$, so a possible pathway for caffeine to interfere with estradiol levels is through a common metabolism. Also, besides caffeine, coffee contains numerous other bioactive substances including lignans and isoflavonoids, both belonging to the phytoestrogen family with great affinity for the estrogen receptor. ${ }^{4}$ As a consequence, changes in hormonal levels might impact on the menstrual cycle, and a daily caffeine consumption $>300 \mathrm{mg}$ has been associated with shorter but not with longer menstrual cycle length. ${ }^{95}$ Moreover, caffeine intake was not associated with the number of oocytes retrieved, the fertilization rate, or implantation rate among women receiving fertility treatment. ${ }^{76}$ The conflicting results on coffee's potential effect on the hormonal pathways are in line with our results suggesting no clear association with fecundity endpoints. In pregnant women, caffeine readily crosses the placental barrier and as a result is distributed to all fetal tissue, making the fetus exposed to the same caffeine levels as the mother. ${ }^{96}$ Also, coffee intake has been associated with decreased levels of both estrogen and $\mathrm{hCG},{ }^{97}$ and caffeine consumption during pregnancy is shown to increase levels of catecholamines and cyclic adenosine monophosphate that might impact on placental blood flow. ${ }^{98-100}$ Indeed, these pathways could have an influence on the increased risk of SAB as observed in this meta-analysis.

This review and meta-analysis has several strengths, including the broader investigation of possible routes of interfering with the ability to conceive and carry a pregnancy to term. Another strength is the dose-response analysis, allowing us to include all information on exposure outcome by including information on intermediate categories, thus making the analysis more effective than the traditional highest versus lowest approach. Using this method, we were able to provide a detailed and more flexible description of the risk of outcome throughout the observed range of exposure. For the SAB analysis, we were able to include a relatively large number of studies providing higher statistical power and allowing us to perform stratified analyses. Hence, we were able to consider potential sources of heterogeneity, including taking into account the varying quality of the included studies.

However, this systematic review and dose-response meta-analysis also has several potential limitations. Though a majority of the included studies adjusted for potential confounders, the presence of residual or unmeasured confounding cannot be excluded due to the observational nature of the included studies. We observed marked between-study 
heterogeneity, which can be caused by methodological or actual differences between the included studies. Consequently, data on SAB were analyzed using a random-effects model. In addition, possible sources of heterogeneity in SAB studies were addressed in stratified analysis, and only study design was found of significance.

Based on the current knowledge, we chose alcohol instead of smoking to be the second most important factor to adjust for in studies reporting on SAB. However, had smoking been selected instead, the NOS would have differed only by one point in three of the 33 evaluated studies as 18 adjusted for both alcohol consumption and smoking, while 12 did not adjust for either of the two. A priori, we determined the threshold for depicting a high-quality study as an NOS $\geq 7$. However, changing the threshold in a sensitivity analysis to NOS $\geq 8$ did not noticeably alter the association in the two strata ( $p$ for heterogeneity $=0.28$ ).

We found evidence of publication bias for studies reporting on $\mathrm{SAB}$, indicating that small studies not reporting any association are less likely to be published. Thus, results from $\mathrm{SAB}$ analysis should be interpreted bearing this in mind. Due to the limited number of studies reporting sufficient data on fecundity or MAR endpoints, assessment of publication bias for these could not be done.

As data on caffeine were self-reported and obtained by various questionnaires or interview, exposure misclassification of coffee/caffeine intake is unavoidable. Especially studies with retrospectively collected exposure data are prone to recall bias. Further, due to the numerous types of questionnaires used to assess exposure, the accuracy and data quality are expected to differ accordingly.

Yet, validation studies support that coffee consumption can be assessed with fairly high validity. ${ }^{101}$ Due to the various sources of exposure, it is difficult to estimate the total caffeine consumption accurately. Further, coffee contains a series of different chemical substances, making it difficult to segregate potential biological effects from caffeine from those of other bioactive compounds. Also, content of the different substances in coffee varies by type of bean, brewing method, and cup size. ${ }^{102}$ Thus, a possible association might be caused by other compounds or by a cocktail effect due to simultaneous exposure to multiple bioactive substances. However, most studies have focused on exposure to caffeine and thus only to some extent on exposure to coffee. In general, exposure assessment was reported as the average daily intake, and consequently we were not able to take the consumption patterns of coffee/caffeine into account. Also, different conversion factors were used in studies when translating coffee consumption into caffeine. In the meta-analysis, we tried to quantify the amount of caffeine intake when exposure was only reported as cups of coffee, but by doing so, we were unable to integrate the pronounced variation in caffeine content by different types of coffee consumed. However, changing the conversion factor up to $150 \mathrm{mg}$ caffeine per cup of coffee in a sensitivity analyses did not change the risk of SAB markedly.

The actual level of caffeine exposure might vary greatly due to individual differences in the CYP1A2 gene activity. ${ }^{70,103}$ Hence, polymorphism of $C Y P 1 A 2$ has been linked to both variability in the clearance rate of caffeine ${ }^{104}$ and variability in serum estradiol concentrations. ${ }^{105}$ As a consequence, the half-life of caffeine varies between 1.5 hours for fast metabolizers and up to 10 hours for slow metabolizers, ${ }^{4}$ making it difficult to settle on a relevant window of exposure.

In the context of $\mathrm{SAB}$, reverse causation has to be considered; a viable pregnancy might produce more pronounced pregnancy symptoms, resulting in greater aversion to coffee and thus a decreased caffeine intake. However, a clinically verified SAB may be diagnosed weeks after the actual fetal demise and thus the yet unrecognized pregnancy loss might be the cause of an increased caffeine consumption rather than the opposite - an increased consumption causing adverse reproductive outcomes. In contrast to previous meta-analyse ${ }^{78,79}$ that presented pooled endpoints of SAB with still birth/fetal death, we only included $\mathrm{SAB}$, as the underlying etiology indeed are different.

Six cohort studies out of the included 32 studies on SAB verified the presence of a pregnancy at the time of reporting exposure, thereby reducing the risk of reversed causation. Among these studies, an association between caffeine intake and SAB was indicated, albeit only statistically significant in three studies.

Due to the great differences in caffeine metabolism, future studies may benefit from actually evaluating circulating caffeine levels or its metabolites (eg, serum paraxanthine, the main metabolite) in linkage with genotyping to heighten the information of the actual exposure level. Thus, future studies could consider Mendelian randomization. Also, to possibly segregate effects of caffeine from those of other bioactive substances in coffee, future studies could consider evaluating the consumption of decaffeinated coffee.

\section{Conclusion}

In conclusion, results from this dose-response meta-analysis support the evidence of an association between coffee/caffeine intake and the risk of SAB. However, results from the $\mathrm{SAB}$ analyses have to be interpreted with caution due to 
the significant heterogeneity and risk of bias detected, not least the considerable risk of publication bias. As coffee is the main source of caffeine consumption, it is still unclear whether the association between coffee/caffeine consumption and SAB is related to the potential harmful effects of caffeine or to some of the other bioactive components of coffee. Viewing the reproductive capacity in a broader perspective, there seems to be little, if any, association between coffee/ caffeine consumption and fecundity. Even so, there is still a need for further studies to investigate the fecundity and MAR endpoints on a larger scale than previously done, using better study designs and including improved reporting combined with relevant adjustment strategies.

\section{Acknowledgments}

$\mathrm{JL}$ is supported by a fully financed $\mathrm{PhD}$-scholarship from Aarhus University. AH is supported by the Danish Diabetes Academy. The Danish Diabetes Academy is funded by the Novo Nordisk Foundation. The funding sources had no involvement in the conduct of this article.

\section{Author contributions}

All authors contributed toward data analysis, drafting and critically revising the paper and agree to be accountable for all aspects of the work.

\section{Disclosure}

The authors report no conflicts of interest in this work.

\section{References}

1. Juul S, Karmaus W, Olsen J. Regional differences in waiting time to pregnancy: pregnancy-based surveys from Denmark, France, Germany, Italy and Sweden. The European Infertility and Subfecundity Study Group. Hum Reprod. 1999;14(5):1250-1254.

2. Schmidt L. Infertility and assisted reproduction in Denmark. Epidemiology and psychosocial consequences. Dan Med Bull. 2006;53:390-417.

3. Jacob MC, McQuillan J, Greil AL. Psychological distress by type of fertility barrier. Hum Reprod. 2007;22(3):885-894.

4. Hermansen K, Bech BH, Dragsted LO, et al. "Kaffe, sundhed og sygdom", Vidensråd for Forebyggelse. [Coffee, health and disease]. 2015 Available from: http://www.vidensraad.dk/sites/default/files/ vidensrad_for_forebyggelse_kaffe_sundhed_og_sygdom_2015.pdf. Accessed November 13, 2017. Danish.

5. EFSA Panel on Dietetic Products, Nutrition and Allergies (NDA). Scientific Opinion on the safety of caffeine. EFSA J. 2015;13(5):4102.

6. WHO. Recommendations on Antenatal Care for a Positive Pregnancy Experience. Geneva: WHO; 2016:152.

7. Bolumar F, Olsen J, Rebagliato M, Bisanti L. Caffeine intake and delayed conception: a European multicenter study on infertility and subfecundity. European Study Group on Infertility Subfecundity. $\mathrm{Am}$ J Epidemiol. 1997;145(4):324-334.

8. Hatch EE, Bracken MB. Association of delayed conception with caffeine consumption. Am J Epidemiol. 1993;138(12):1082-1092.
9. Olsen J. Cigarette smoking, tea and coffee drinking, and subfecundity. Am J Epidemiol. 1991;133(7):734-739.

10. Alderete E, Eskenazi B, Sholtz R. Effect of cigarette smoking and coffee drinking on time to conception. Epidemiology. 1995;6(4):403-408.

11. Joesoef MR, Beral V, Rolfs RT, Aral SO, Cramer DW. Are caffeinated beverages risk factors for delayed conception? Lancet. 1990;335(8682):136-137.

12. Curtis KM, Savitz DA, Arbuckle TE. Effects of cigarette smoking, caffeine consumption, and alcohol intake on fecundability. 1997;146:32-41.

13. Christianson RE, Oechsli FW, Berg BJ. Caffeinated beverages and decreased fertility. Lancet. 1989;1(8634):378.

14. Jensen TK, Henriksen TB, Hjollund NH, et al. Caffeine intake and fecundability: a follow-up study among 430 Danish couples planning their first pregnancy. Reprod Toxicol. 1998;12(3):289-295.

15. Wilcox A, Weinberg C, Baird D. Caffeinated beverages and decreased fertility. Lancet. 1988;2(8626-8627):1453-1456.

16. Caan B, Quesenberry CP, Jr, Coates AO. Differences in fertility associated with caffeinated beverage consumption. Am J Public Health. 1998;88(2):270-274.

17. Florack EI, Zielhuis GA, Rolland R. Cigarette smoking, alcohol consumption, and caffeine intake and fecundability. Prev Med. 1994;23(2):175-180.

18. Spinelli A, Figa-Talamanca I, Osborn J. Time to pregnancy and occupation in a group of Italian women. Int J Epidemiol. 1997;26(3):601-609.

19. Hakim RB, Gray RH, Zacur H. Alcohol and caffeine consumption and decreased fertility. Fertil Steril. 1998;70:632-637.

20. Matijasevich A, Barros FC, Santos IS, Yemini A. Maternal caffeine consumption and fetal death: a case-control study in Uruguay. Paediatr Perinat Epidemiol. 2006;20(2):100-109.

21. Bech BH, Nohr EA, Vaeth M, Henriksen TB, Olsen J. Coffee and fetal death: a cohort study with prospective data. Am J Epidemiol. 2005;162(10):983-990.

22. Wisborg K, Kesmodel U, Bech BH, Hedegaard M, Henriksen TB. Maternal consumption of coffee during pregnancy and stillbirth and infant death in first year of life: prospective study. $B M J$. 2003;326(7386):420.

23. Greenwood DC, Thatcher NJ, Ye J, et al. Caffeine intake during pregnancy and adverse birth outcomes: a systematic review and doseresponse meta-analysis. Eur J Epidemiol. 2014;29(10):725-734.

24. Maslova E, Bhattacharya S, Lin SW, Michels KB. Caffeine consumption during pregnancy and risk of preterm birth: a meta-analysis. $\mathrm{Am}$ J Clin Nutr. 2010;92(5):1120-1132.

25. PROSPERO. International prospective register of systematic reviews. 2017. Available from: https://www.crd.york.ac.uk/PROSPERO/. Accessed November 13, 2017.

26. Wells GA, Shea B, O'Connell D, et alNewcastle-Ottawa Scale (NOS); 2017. Available from: http://www.ohri.ca/programs/clinical_epidemiology/oxford.asp. Accessed May 8, 2017.

27. Liberati A, Altman DG, Tetzlaff J, et al. The PRISMA statement for reporting systematic reviews and meta-analyses of studies that evaluate health care interventions: explanation and elaboration. Ann Intern Med. 2009;151(4):W65-W94.

28. Wilcox A. Fertility and Pregnancy. New York, NY, Oxford University Press; 2010.

29. Pollack AZ, Buck Louis GM, Sundaram R, Lum KJ. Caffeine consumption and miscarriage: a prospective cohort study. Fertil Steril. 2010;93(1):304-306.

30. Taylor KC, Small CM, Dominguez CE, et al. Alcohol, smoking, and caffeine in relation to fecundability, with effect modification by NAT2. Ann Epidemiol. 2011;21(11):864-872.

31. Axelsson G, Rylander R. Outcome of pregnancy in women engaged in laboratory work at a petrochemical plant. Am J Ind Med. 1989;16(5):539-545.

32. Cnattingius S, Signorello LB, Anneren G, et al. Caffeine intake and the risk of first-trimester spontaneous abortion. $N$ Engl $J$ Med. 2000;343(25):1839-1845. 
33. Stefanidou EM, Caramellino L, Patriarca A, Menato G. Maternal caffeine consumption and sine causa recurrent miscarriage. Eur J Obstet Gynecol Reprod Biol. 2011;158(2):220-224.

34. Srisuphan WF, Bracken MB. Caffeine consumption during pregnancy and association with late spontaneous abortion. 1986;154(1):14-20.

35. Khoury JC, Miodovnik M, Buncher CR, et al. Consequences of smoking and caffeine consumption during pregnancy in women with type 1 diabetes. J Matern Fetal Neonatal Med. 2004;15(1):44-50.

36. Parazzini F, Bocciolone L, Fedele L, Negri E, La Vecchia C, Acaia B. Risk factors for spontaneous abortion. Int $J$ Epidemiol. 1991;20(1):157-161.

37. Lubna A, Al-Ansary ZAB. Risk factors for spontaneous abortion: a preliminary study on Saudi women. J Roy Soc Health. 1994;114:188-193.

38. Orsini N, Li R, Wolk A, Khudyakov P, Spiegelman D. Meta-analysis for linear and nonlinear dose-response relations: examples, an evaluation of approximations, and software. Am J Epidemiol. 2012;175(1):66-73.

39. Crippa AO, N. Dose-response meta-analysis of differences in means. BMC Med Res Methodol. 2016;16:91.

40. Chen DG, Fang D, Wilson JR. Meta-analysis of two studies with random effects? J Minim Invasive Gynecol. 2017;24(5):689-690.

41. McCusker RR, Goldberger BA, Cone EJ. Caffeine content of specialty coffees. J Anal Toxicol. 2003;27(7):520-522.

42. Greenland S. Dose-response and trend analysis in epidemiology: alternatives to categorical analysis. Epidemiology. 1995;6(4):356-365.

43. Durrleman S, Simon R. Flexible regression models with cubic splines. Stat Med. 1989;8(5):551-561.

44. Desquilbet L, Mariotti F. Dose-response analyses using restricted cubic spline functions in public health research. Stat Med. 2010;29(9):1037-1057.

45. Higgins JP, Thompson SG, Deeks JJ, Altman DG. Measuring inconsistency in meta-analyses. BMJ. 2003;327(7414):557-560.

46. Egger M, Davey Smith G, Schneider M, Minder C. Bias in meta-analysis detected by a simple, graphical test. BMJ. 1997;315(7109):629-634.

47. Green JPHaS. Cochrane Handbook for Systematic Reviews of Interventions; 2017. Available from: http://handbook.cochrane. org/chapter_10/10_4_3_1_recommendations_on_testing_for_funnel_plot_asymmetry.htm. Accessed June 2, 2017.

48. R Core Team. R: A language and environment for statistical computing; 2009. Available from: https://www.r-project.org. Accessed November 13, 2017.

49. Viechtbauer W. Conducting meta-analyses in $\mathrm{R}$ with metafor package. J Stat Softw. 2010;36(3):48.

50. Hatch EE, Wise LA, Mikkelsen EM, et al. Caffeinated beverage and soda consumption and time to pregnancy. Epidemiology. 2012;23(3):393-401.

51. Fenster L, Eskenazi B, Windham GC, Swan SH. Caffeine consumption during pregnancy and spontaneous abortion. Epidemiology. 1991;2(3):168-174.

52. Fenster L, Hubbard AE, Swan SH, et al. Caffeinated beverages, decaffeinated coffee, and spontaneous abortion. Epidemiology. 1997;8(5):515-523.

53. Armstrong BG, McDonald AD, Sloan M. Cigarette, alcohol, and coffee consumption and spontaneous abortion. Am J Public Health. 1992;82(1):85-87.

54. Dominguez-Rojas V, de Juanes-Pardo R, Astasio-Arbiza P, OrtegaMolina P, Gordillo-Florencio E. Spontaneous abortion in a hospital population: are tobacco and coffee intake risk factors? Eur J Epidemiol. 1994;10(6):665-668

55. Dlugosz L, Belanger K, Hellenbrand K, Holford TR, Leaderer B, Bracken MB. Maternal caffeine consumption and spontaneous abortion: a prospective cohort study. Epidemiology. 1996;7(3):250-255.

56. Agnesi R, Valentini F, Fedeli U, et al. Maternal exposures and risk of spontaneous abortion before and after a community oriented health education campaign. Eur J Public Health. 2010;21(3):282-285.

57. Agnesi R, Valentini F, Mastrangelo G. Risk of spontaneous abortion and maternal exposure to organic solvents in the shoe industry. Int Arch Occup Environ Health. 1997;69(5):311-316.
58. Giannelli M, Doyle P, Roman E, Pelerin M, Hermon C. The effect of caffeine consumption and nausea on the risk of miscarriage. Paediatr Perinat Epidemiol. 2003;17(4):316-323.

59. George L, Granath F, Johansson AL, Olander B, Cnattingius S. Risks of repeated miscarriage. Paediatr Perinat Epidemiol. 2006;20(2):119-126.

60. Kline J, Levin B, Silverman J, et al. Caffeine and spontaneous abortion of known karyotype. Epidemiology. 1991;2(6):409-417.

61. Infante-Rivard C, Fernandez A, Gauthier R, David M, Rivard, GE. Fetal loss associated with caffeine intake before and during pregnancy. JAMA. 1993;270(24);2940-2943.

62. Mills JL, Holmes LB, Aarons JH, et al. Moderate caffeine use and the risk of spontaneous-abortion and intrauterine growth-retardation. JAMA. 1993;269(5):593-597.

63. Parazzini F, Chatenoud L, Di Cintio E, et al. Coffee consumption and risk of hospitalized miscarriage before 12 weeks of gestation. Hum Reprod. 1998;13(8):2286-2291.

64. Maconochie N, Doyle P, Prior S, Simmons R. Risk factors for first trimester miscarriage - results from a UK-population-based casecontrol study. BJOG. 2007;114(2):170-186.

65. Greenwood DC, Alwan N, Boylan S, et al. Caffeine intake during pregnancy, late miscarriage and stillbirth. Eur J Epidemiol. 2010;25(4):275-280.

66. Wilcox AJ, Weinberg CR, Baird DD. Risk factors for early pregnancy loss. Epidemiology. 1990;1(5):382-385.

67. Zhang H, Bracken MB. Tree-based, two-stage risk factor analysis for spontaneous abortion. Am J Epidemiol. 1996;144(10):989-996.

68. Wen W, Shu XO, Jacobs DR, Jr, Brown JE. The associations of maternal caffeine consumption and nausea with spontaneous abortion. Epidemiology. 2001;12(1):38-42.

69. Tolstrup JS, Munk C, Madsen LB, Ottesen B, Bergholt T, Gronbaek M. Does caffeine and alcohol intake before pregnancy predict the occurrence of spontaneous abortion? Hum Reprod. 2003;18(12):2704-2710.

70. Sata F, Yamada H, Suzuki K, et al. Caffeine intake, CYP1A2 polymorphism and the risk of recurrent pregnancy loss. Mol Hum Reprod. 2005;11(5):357-360.

71. Savitz DA, Chan RL, Herring AH, Howards PP, Hartmann KE. Caffeine and miscarriage risk. Epidemiology. 2008;19(1):55-62.

72. Weng X, Odouli R, Li DK. Maternal caffeine consumption during pregnancy and the risk of miscarriage: a prospective cohort study. $\mathrm{Am}$ J Obstet Gynecol. 2008;198(3):279.e1-8.

73. Hahn KA, Wise LA, Rothman KJ, et al. Caffeine and caffeinated beverage consumption and risk of spontaneous abortion. Hum Reprod. 2015;30(5):1246-1255.

74. Klonoff-Cohen H, Bleha J, Lam-Kruglick P. A prospective study of the effects of female and male caffeine consumption on the reproductive endpoints of IVF and gamete intra-Fallopian transfer. Hum Reprod. 2002;17(7):1746-1754.

75. Al-Saleh I, El-Doush I, Grisellhi B, Coskun S. The effect of caffeine consumption on the success rate of pregnancy as well various performance parameters of in-vitro fertilization treatment. Med Sci Monit. 2010;16(12):CR598-CR605.

76. Choi JH, Ryan LM, Cramer DW, Hornstein MD, Missmer SA. Effects of caffeine consumption by women and men on the outcome of in vitro fertilization. J Caffeine Res. 2011;1(1):29-34.

77. Rasch V. Cigarette, alcohol, and caffeine consumption: risk factors for spontaneous abortion. Acta Obstet Gynecol Scand. 2003;82(2):182-188.

78. Li J, Zhao H, Song JM, Zhang J, Tang YL, Xin CM. A meta-analysis of risk of pregnancy loss and caffeine and coffee consumption during pregnancy. Int J Gynaecol Obstet. 2015;130(2):116-122.

79. Chen LW, Wu Y, Neelakantan N, Chong MF, Pan A, van Dam RM. Maternal caffeine intake during pregnancy and risk of pregnancy loss: a categorical and dose-response meta-analysis of prospective studies. Public Health Nutr. 2016;19(7):1233-1244.

80. Fredholm BB. On the mechanism of action of theophylline and caffeine. Acta Med Scand. 1985;217(2):149-153.

81. Soyka LF. Effects of methylxanthines on the fetus. Clin Perinatol. 1979;6(1):37-51. 
82. Bellet S, Roman L, DeCastro O, Kim KE, Kershbaum A. Effect of coffee ingestion on catecholamine release. Metabolism. 1969;18(4):288-291.

83. London S, Willett W, Longcope C, McKinlay S. Alcohol and other dietary factors in relation to serum hormone concentrations in women at climacteric. Am J Clin Nutr. 1991;53(1):166-171.

84. Petridou E, Katsouyanni K, Spanos E, Skalkidis Y, Panagiotopoulou K, Trichopoulos D. Pregnancy estrogens in relation to coffee and alcohol intake. Ann Epidemiol. 1992;2(3):241-247.

85. Kotsopoulos J, Eliassen AH, Missmer SA, Hankinson SE, Tworoger SS. Relationship between caffeine intake and plasma sex hormone concentrations in premenopausal and postmenopausal women. Cancer. 2009; 115(12):2765-2774.

86. Schliep KC, Schisterman EF, Mumford SL, et al. Caffeinated beverage intake and reproductive hormones among premenopausal women in the BioCycle Study. Am J Clin Nutr. 2012;95(2):488-497.

87. Goto A, Song Y, Chen BH, Manson JE, Buring JE, Liu S. Coffee and caffeine consumption in relation to sex hormone-binding globulin and risk of type 2 diabetes in postmenopausal women. Diabetes. 2011;60(1):269-275.

88. Nagata C, Kabuto M, Shimizu H. Association of coffee, green tea, and caffeine intakes with serum concentrations of estradiol and sex hormone-binding globulin in premenopausal Japanese women. Nutr Cancer. 1998;30(1):21-24.

89. Ferrini RL, Barrett-Connor E. Caffeine intake and endogenous sex steroid levels in postmenopausal women. The Rancho Bernardo Study Am J Epidemiol. 1996;144(7):642-644.

90. Lucero J, Harlow BL, Barbieri RL, Sluss P, Cramer DW. Early follicular phase hormone levels in relation to patterns of alcohol, tobacco, and coffee use. Fertil Steril. 2001;76(4):723-729.

91. Kinney A, Kline J, Kelly A, Reuss ML, Levin B. Smoking, alcohol and caffeine in relation to ovarian age during the reproductive years. Hum Reprod. 2007;22(4):1175-1185.

92. Cooper C, Atkinson EJ, Wahner HW, et al. Is caffeine consumption a risk factor for osteoporosis? J Bone Miner Res. 1992;7(4):465-471.

93. Butler MA, Iwasaki M, Guengerich FP, Kadlubar FF. Human cytochrome $\mathrm{P}-450 \mathrm{PA}$ (P-450IA2), the phenacetin O-deethylase, is primarily responsible for the hepatic 3-demethylation of caffeine and N-oxidation of carcinogenic arylamines. Proc Natl Acad Sci USA. 1989;86(20):7696-7700.
94. Yamazaki H, Shaw PM, Guengerich FP, Shimada T. Roles of cytochromes P450 1A2 and 3A4 in the oxidation of estradiol and estrone in human liver microsomes. Chem Res Toxicol. 1998;11(6): 659-665.

95. Fenster L, Quale C, Waller K, et al. Caffeine consumption and menstrual function. Am J Epidemiol. 1999;149(6):550-557.

96. Aldridge A, Bailey J, Neims AH. The disposition of caffeine during and after pregnancy. Semin Perinatol. 1981;5(4):310-314.

97. Lawson CC, LeMasters GK, Levin LS, Liu JH. Pregnancy hormone metabolite patterns, pregnancy symptoms, and coffee consumption. Am J Epidemiol. 2002;156(5):428-437.

98. Kirkinen P, Jouppila P, Koivula A, Vuori J, Puukka M. The effect of caffeine on placental and fetal blood flow in human pregnancy. Am J Obstet Gynecol. 1983;147(8):939-942.

99. Weathersbee PS, Lodge JR. Caffeine: its direct and indirect influence on reproduction. J Reprod Med. 1977;19(2):55-63.

100. Morris MB, Weinstein L. Caffeine and the fetus: is trouble brewing? Am J Obstet Gynecol. 1981;140(6):607-610.

101. Ferraroni M, Tavani A, Decarli A, et al. Reproducibility and validity of coffee and tea consumption in Italy. Eur J Clin Nutr. 2004;58(4):674-680.

102. Bracken MB, Triche E, Grosso L, Hellenbrand K, Belanger K, Leaderer BP. Heterogeneity in assessing self-reports of caffeine exposure: implications for studies of health effects. Epidemiology. 2002;13(2):165-171.

103. Grosso LM, Bracken MB. Caffeine metabolism, genetics, and perinatal outcomes: a review of exposure assessment considerations during pregnancy. Ann Epidemiol. 2005;15(6):460-466.

104. Sachse C, Brockmoller J, Bauer S, Roots I. Functional significance of a $\mathrm{C}-->\mathrm{A}$ polymorphism in intron 1 of the cytochrome $\mathrm{P} 450$ CYP1A2 gene tested with caffeine. Br J Clin Pharmacol. 1999;47(4): 445-449.

105. Lurie G, Maskarinec G, Kaaks R, Stanczyk FZ, Le Marchand L. Association of genetic polymorphisms with serum estrogens measured multiple times during a 2-year period in premenopausal women. Cancer Epidemiol Biomarkers Prev. 2005;14(6):1521-1527.
Clinical Epidemiology

\section{Publish your work in this journal}

Clinical Epidemiology is an international, peer-reviewed, open access, online journal focusing on disease and drug epidemiology, identification of risk factors and screening procedures to develop optimal preventative initiatives and programs. Specific topics include: diagnosis, prognosis, treatment, screening, prevention, risk factor modification, systematic reviews, risk and safety of medical interventions, epidemiology and biostatistical methods, and evaluation of guidelines, translational medicine, health policies and economic evaluations. The manuscript management system is completely online and includes a very quick and fair peer-review system, which is all easy to use. 